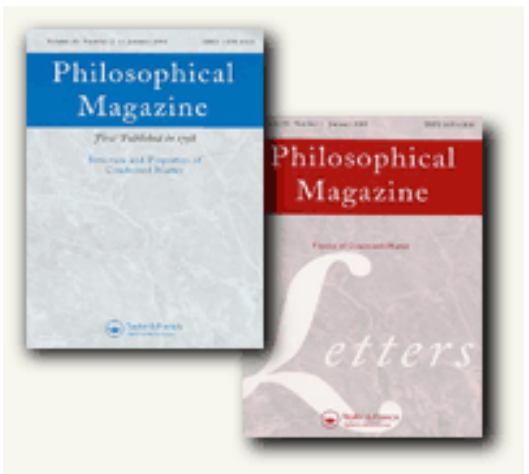

\title{
Numerical Implementation of a 3D Continuum Theory of Dislocation Dynamics and Application to Microbending
}

\begin{tabular}{|r|l|}
\hline Journal: & Philosophical Magazine \& Philosophical Magazine Letters \\
\hline Manuscript ID: & TPHM-09-Mar-0134.R2 \\
\hline Journal Selection: & Philosophical Magazine \\
\hline Author: & O2-Aug-2009 \\
\hline & $\begin{array}{l}\text { Complete List of Authors: } \\
\text { IWF } \\
\text { Gumbschrainer, Peter; University of Karlsruhe, Institute for Reliability of } \\
\text { Components and Systems; Fraunhofer-Institut fuer } \\
\text { Werkstoffmechanik IWF } \\
\text { Zaiser, Michael; The University of Edinburgh, Institute for Materials } \\
\text { and Processes }\end{array}$ \\
\hline \hline Keywords: & $\begin{array}{l}\text { constitutive equations, dislocation dynamics, dislocation theory, } \\
\text { plasticity of crystals }\end{array}$ \\
\hline Keywords (user supplied): & constitutive equations, dislocation dynamics, dislocation theory \\
\hline
\end{tabular}

\section{scholarONE" \\ Manuscript Central}




\title{
Numerical Implementation of a 3D Continuum Theory of Dislocation Dynamics and Application to Microbending
}

\author{
S. Sandfeld ${ }^{a, c} *$, T. Hochrainer ${ }^{\mathrm{a}, \mathrm{b}}$, P. Gumbsch ${ }^{\mathrm{a}, \mathrm{b}}$, M. Zaiser ${ }^{\mathrm{c}}$ \\ ${ }^{a}$ Institut für Zuverlässigkeit von Bauteilen und Systemen, \\ Universität Karlsruhe (TH), Kaiserstr. 12, 76131 Karlsruhe, Germany; \\ ${ }^{\mathrm{b}}$ Fraunhofer-Institut für Werkstoffmechanik IWM, \\ Wöhlerstr. 11, 79108 Freiburg, Germany; \\ ${ }^{\mathrm{c}}$ The University of Edinburgh, Institute for Materials and Processes, \\ The Kings Buildings, Sanderson Building, Edinburgh EH9 3JL, United Kingdom
}

(April 2009)

\begin{abstract}
Crystal plasticity is governed by the motion of lattice dislocations. Although continuum theories of static dislocation assemblies date back to the 1950's, the line-like character of these defects posed serious problems for the development of a continuum theory of plasticity which is based on the averaged dynamics of dislocation systems. Only recently the geometrical problem of performing meaningful averages over systems of moving, oriented lines has been solved. Such averaging leads to the definition of a dislocation density tensor of second order along with its evolution equation. This tensor can be envisaged as the analogue of the classical dislocation density tensor in an extended space which includes the line orientation as an independent variable. In the current work we discuss the numerical implementation of a continuum theory of dislocation evolution that is based on this dislocation density measure and apply this to some simple benchmark problems as well as to plane-strain microbending.
\end{abstract}

Keywords: dislocation dynamics; continuum theory of dislocation; dislocation density; microbending

\section{Introduction}

In recent years, the growing demand for physically motivated continuum descriptions of plasticity has led to a renewed effort to formulate continuum theories of dislocation kinematics and dynamics. Already in the 1950s, Kröner [1], Nye [2], Bilby and co-workers [3] and Kondo [4] independently formulated the classical continuum theory of dislocations based upon the definition of a second-rank dislocation density tensor (in the following denoted as Kröner-Nye tensor). The authors were well aware that the Kröner-Nye tensor, if used as a measure of the average dislocation state of a crystal, leads to a description of plastic deformation processes that is intrinsically incomplete. The main problem with classical dislocation density measures such as the Kröner-Nye tensor is that its averaging over a volume element containing many dislocation lines produces a measure that is invariant upon shear deformations of the averaging volume. As a consequence, any spatially homogeneous shear deformation can in principle not be reflected by the dynamic evolution of the Kröner-Nye tensor or similar measures. This renders the classical dislocation density measures highly problematic as foundations for a continuum theory of plasticity.

*Corresponding author. Email: stefan.sandfeld@kit.edu 
Nevertheless, plasticity theories have been formulated which are explicitly or implicitly based upon the classical dislocation density measures. As we shall discuss in detail, such theories, as for instance formulated by Acharya and co-workers [5], [6], [7] and by Sedláček et al. [8], apply to situations where dislocations are forming smooth bundles, i.e. adjacent dislocation lines are approximately parallel and have the same orientation. In more general situations, using the classical dislocation density tensor as the basis of a plasticity theory leads to non-closed formulations which need to be 'patched up' by phenomenological assumptions. Models which focus on the evolution of scalar densities of so-called statistically stored and geometrically neccessary dislocations (see e.g. [9] and references therein) are also essentially phenomenological. Such models can be considered as heuristic approaches towards formulating constitutive equations which allude to the physical mechanisms of dislocation microstructure evolution. However, the constitutive equations are not related to the evolution of a discrete dislocation system through systematic and mathematically meaningful averaging procedures, and the models therefore remain on the level of phenomenological expedients [10].

From the late 1990s on, Groma and co-workers developed a statistical approach towards deriving not only the kinematic evolution of dislocation systems, but also their internal interactions and stress driven dynamics, from systematic averages over ensembles of discrete dislocation systems [11], [12], [13]. This approach draws heavily on averaging techniques used in the statistical mechanics of interacting particle systems. It is therefore restricted to simplified systems of straight edge dislocations, which can be imagined as two-dimensional systems of point particles in the intersecting plane. In an average representation, these systems can be described in terms of densities of charged particles ('positive' and 'negative' dislocations distinguished by the two possible orientations of the line direction with respect to the Burgers vector). It is not obvious how to generalise these methods to threedimensional systems of connected dislocation lines, since the line-like nature of the dislocations and the continuous distribution of dislocation line directions need to be accounted for.

A description of dislocation systems in terms of higher-dimensional density measures dates back to Kosevich [14], who defines dislocation densities in a space which includes parameters characterising the line orientation as independent variable. In the context of dislocation dynamics, a similar approach was used in El-Azab's pioneering work [15], who extended the methods of statistical mechanics to systems of curved dislocation lines by using densities that evolve in a higher dimensional state space. However, while taking into account the orientation of line segments, both Kosevich and El-Azab do not directly account for the local connectivity of the segments. To illustrate the problem, we observe that their measures are not able to distinguish between a distribution of circular loops and a distribution of randomly oriented straight dislocation lines in a plane. The kinematic properties of these two systems are, however, very different.

Only recently, the mathematical foundations required for transferring the methods of statistical mechanics consistently to three-dimensional systems of curved dislocations have been formulated by Hochrainer's 'Extended Continuum Theory' (ECT) [16]. This theory relies on a geometrical description of dislocation lines and their averages, i.e. line densities, which uses the methods and formalisms of differential geometry. The theory is a direct generalization of Kröner's classical continuum theory, which is contained as a special case.

In this paper, we explore ECT from the point of view of its numerical implementation for a single slip geometry. Section 2 presents the theoretical background and is divided into three parts: section 2.1 briefly explains the constitutive relations 


\section{Page 3 of 30 \\ pm-paper-revised-v1}

governing the stress-strain response of a (macroscopic) solid. For completeness, we then introduce in section 2.2 Kröner's classical continuum theory and afterwards provide an overview of some of the theoretical aspects and basic notations of ECT in section 2.3. After discussing basic ideas of the numerical implementation in section 3, we explore in section 4 the evolution equations numerically and apply them to a continuous distribution of dislocation loops and, as a benchmark for kinematic consistency, to the expansion of a quasi-isolated single dislocation loop. In section 5 we then apply the theory to analyze the dislocation evolution during bending of a free-standing thin film and investigate the size effects associated with a simple Taylor-type hardening model. Conclusions are presented in section 6 .

Throughout the paper we use the following definitions and conventions: vectors and tensors are denoted by bold-face letters, whereas scalar quantities are written non-bold (e.g. $\boldsymbol{\alpha}$ as opposed to $\alpha$ ). Partial derivatives are abbreviated as $\partial_{x}(\cdot):=\frac{\partial(\cdot)}{\partial x}, \nabla$ denotes the gradient operator and the divergence of a vector (tensor) field is written as div; curl denotes the curl-operator. The vector product is denoted by $\times$. For double indices we assume the summation convention, if not stated otherwise. In $\boldsymbol{c}=\boldsymbol{a} \otimes \boldsymbol{b}$ the tensor product is denoted by $\otimes$; this operation reads for cartesian coordinates in index notation $c_{i j}=a_{i} b_{j}$. The symmetric part of a second rank tensor $\boldsymbol{a}$ can be obtained by sym $\boldsymbol{a}:=\frac{1}{2}\left(\boldsymbol{a}+\boldsymbol{a}^{T}\right)$ with $\boldsymbol{a}^{T}$ denoting the transpose of a matrix.

\section{Theoretical Background}

\subsection{Small strain elasto-plasticity}

In continuum mechanics the deformation of a body is usually characterised by a smooth vector field of displacements $\boldsymbol{u}$. The gradient $\nabla \boldsymbol{u}$ is called the distortion tensor and is in the following denoted by $\boldsymbol{\beta}$ :

$$
\boldsymbol{\beta}:=\nabla \boldsymbol{u}
$$

The symmetric part of the distortion tensor is called the strain tensor:

$$
\varepsilon:=\operatorname{sym} \boldsymbol{\beta}=\operatorname{sym} \nabla \boldsymbol{u} .
$$

In small-strain elasto-plasticity the distortion tensor is additively decomposed into a stress-free or plastic part $\boldsymbol{\beta}^{p l}$ and an elastic part $\boldsymbol{\beta}^{e l}$. Accordingly, the strain is additively decomposed as

$$
\varepsilon=\varepsilon^{e l}+\varepsilon^{p l}=\operatorname{sym} \boldsymbol{\beta}^{e l}+\operatorname{sym} \boldsymbol{\beta}^{p l} .
$$

The local stress $\boldsymbol{\sigma}$ is related to the elastic part of the strain tensor by the consitutive law

$$
\boldsymbol{\sigma}=\mathbb{C}: \varepsilon^{e l}=\mathbb{C}:\left(\varepsilon-\varepsilon^{p l}\right),
$$

where $\mathbb{C}$ denotes the fourth order tensor of elastic moduli and ':' the doubly contracted tensor product, in Cartesian coordinates $\sigma_{i j}=C_{i j k l} \varepsilon_{k l}^{e l}$. The last equation is accompanied by balance equations for momentum and angular momentum, which 
in the absence of body and inertial forces read

$$
\operatorname{div} \boldsymbol{\sigma}=\mathbf{0} \text { and } \boldsymbol{\sigma}=\boldsymbol{\sigma}^{\mathbf{T}}
$$

respectively.

The purpose of constitutive modelling in plasticity theory is to derive evolution equations for the plastic distortion $\boldsymbol{\beta}^{p l}$ based on the current stress state and possibly various internal variables of the material. ECT is an example of a theory where the evolution of the distortion $\boldsymbol{\beta}^{p l}$ is expressed in terms of internal variables which represent statistical averages over the discrete dislocation pattern within the crystal.

\subsection{Classical continuum theory of dislocations}

Kröner [1] defined the dislocation density tensor $\boldsymbol{\alpha}$ as

$$
\boldsymbol{\alpha}=\operatorname{curl} \boldsymbol{\beta}^{p l}
$$

A different but equivalent definition was given by Nye [2]. In Eq. (6), the curl operator acts on the first indices of a tensor in the same way as it acts on a vector. From the definition of the Kröner-Nye tensor it immediately follows that

$$
\operatorname{div} \boldsymbol{\alpha}=0
$$

which reflects the physical fact that dislocations cannot end inside a crystal.

When analyzing the information about the dislocation system that is contained in the Kröner-Nye tensor, it is important to specify the spatial resolution with which the plastic distortion is described. This is not always recognized in the literature, as the very concept of 'spatial resolution' seems odd when we are talking about a continuum theory. However, in order to understand the strengths and limitations of the classical continuum theory of dislocations it is indispensable to analyze how the information content expressed by the fundamental dislocation density measure changes as we move across scales. We start this analysis on the smallest scale on which a continuum theory can be used, i.e., we use continuous fields to describe the distortion of the crystal but assume that these fields are known with sufficient resolution such that individual dislocations can be 'seen' as singular lines.

\subsubsection{Dislocation density tensor for a discrete dislocation system}

If the spatial resolution of the theory is such that all dislocation lines are captured separately by the curl operation, the Kröner-Nye tensor completely characterises the dislocation system. In this case $\boldsymbol{\alpha}$ can be explicitly related to the configuration of the discrete dislocation lines. We envisage a dislocation line as an oriented curve $\boldsymbol{c}(s)$ which we assume to be parametrised by $\operatorname{arc}$ length $s$, i.e. $\mathrm{d} \boldsymbol{c} / \mathrm{d} s$ is the unit tangent vector to the dislocation line. Furthermore we assume that all dislocations share the same Burgers vector $\boldsymbol{b}$. In situations where dislocations of several slip systems are present, the following considerations hold for each slip system separately, and the total dislocation density tensor is obtained by summing over all slip systems. 


\section{Page 5 of 30 \\ Philosophical Magazine \& Philosophical Magazine Letters}

Philosophical Magazine

We formally define a density measure along the dislocation line by

$$
\delta_{\boldsymbol{c}}(\boldsymbol{r})=\int_{0}^{L_{\mathrm{c}}} \delta(\boldsymbol{c}(s)-\boldsymbol{r}) \mathrm{d} s,
$$

where $L_{\boldsymbol{c}}$ is the total length of curve $\boldsymbol{c}$ and $\delta(\boldsymbol{r})$ is the standard Dirac measure ('delta function') in three-dimensional space. Using this measure, we may write the discrete (indicated by superscript 'd') Kröner-Nye tensor as

$$
\boldsymbol{\alpha}^{d}=\sum_{\boldsymbol{c}} \delta_{\boldsymbol{c}} \frac{\mathrm{d} \boldsymbol{c}}{\mathrm{d} s} \otimes \boldsymbol{b}
$$

where the sum runs over all dislocation lines in the system. The local rate of plastic distortion is given by Orowan's relation which in tensorial form reads

$$
\partial_{t} \boldsymbol{\beta}^{p l, d}=-\sum_{\boldsymbol{c}} \delta_{\boldsymbol{c}} v \boldsymbol{n} \otimes \boldsymbol{b}=-\boldsymbol{v} \times \boldsymbol{\alpha}^{d} .
$$

Here, $\boldsymbol{v}=v \boldsymbol{\nu}$ is the local dislocation velocity, with $v$ being the scalar velocity, and $\boldsymbol{\nu}$ the unit vector in the dislocation glide direction. In writing down (10), we used that the glide plane normal is given by $\boldsymbol{n}=\boldsymbol{\nu} \times \frac{\mathrm{d} \boldsymbol{c}}{\mathrm{d} s}$.

Defining the discrete dislocation current by $\boldsymbol{J}^{d}=\boldsymbol{v} \times \boldsymbol{\alpha}^{d}$ and using the definition of the Kröner-Nye tensor yields a kinematically closed evolution equation ${ }^{1}$ for $\boldsymbol{\alpha}^{d}$ :

$$
\partial_{t} \boldsymbol{\alpha}^{d}=-\operatorname{curl} \boldsymbol{J}^{d}=-\operatorname{curl}\left(\boldsymbol{v} \times \boldsymbol{\alpha}^{d}\right) .
$$

We now attempt to use these relations for constructing a coarse-grained theory. In doing so, we ask whether or not (and under which circumstances) direct coarse graining of the classical dislocation density measure leads to a loss of essential information.

\subsubsection{Statistical averaging}

We denote by $\langle(\ldots)\rangle_{V, \boldsymbol{r}}:=(1 / V) \int_{V_{\boldsymbol{r}}}(\ldots) \mathrm{d}^{3} r$ the spatial average over some small volume $V_{\boldsymbol{r}}$ of size $V$ centered at $\boldsymbol{r}$ (for convenience of notation, the subscripts $V$ and $\boldsymbol{r}$ will be dropped in the following). A scalar measure of the average dislocation density is given by the dislocation line length within $V_{\boldsymbol{r}}$, divided by the averaging volume:

$$
\begin{aligned}
\rho & =\frac{1}{V} \sum_{\boldsymbol{c}} \int_{\boldsymbol{c} \cap V} 1 \mathrm{~d} s \\
& =\frac{1}{V} \sum_{\boldsymbol{c}} \int_{V} \int_{0}^{L_{\boldsymbol{c}}} \delta\left(\boldsymbol{c}(s)-\boldsymbol{r}^{\prime}\right) \mathrm{d}^{3} r^{\prime} \mathrm{d} s=\left\langle\sum_{\boldsymbol{c}} \delta_{c}\right\rangle .
\end{aligned}
$$

\footnotetext{
${ }^{1}$ We speak of a kinematically closed equation as we assume the dislocation velocity $v$ at this point to be a given quantity. In general, $v$ is a function of the local stress, which in turn depends on the dislocation arrangement. Hence, a mathematically closed theory requires additional relationships between the dislocation state, as expressed by $\alpha$, and the dislocation velocity $v$. If $v$ is a function of stress and line direction only, these relationships may be derived from Kröner's theory of eigenstresses [1].
} 
This measure characterises the total dislocation density. Similarly, we define the average dislocation density tensor by

$$
\boldsymbol{\alpha}=\left\langle\boldsymbol{\alpha}^{d}\right\rangle=\left\langle\sum_{c} \delta_{c} \frac{\mathrm{d} \boldsymbol{c}}{\mathrm{d} s} \otimes \boldsymbol{b}\right\rangle,
$$

The average line direction is given by the unit vector

$$
l=\frac{\left\langle\sum_{\boldsymbol{c}} \delta_{\boldsymbol{c}} \frac{\mathrm{d} \boldsymbol{c}}{\mathrm{d} s}\right\rangle}{\|\left\langle\sum_{\boldsymbol{c}} \delta_{\boldsymbol{c}} \frac{\mathrm{d} \boldsymbol{d}}{\mathrm{d} s}\right\rangle},
$$

and we define the geometrically necessary dislocation density $\rho_{\mathrm{G}}$ by

$$
\rho_{\mathrm{G}}=\left\|\left\langle\sum_{c} \delta_{c} \frac{\mathrm{d} c}{\mathrm{~d} s}\right\rangle\right\| .
$$

The ratio $\rho_{\mathrm{G}} / \rho$ is always smaller equal than 1 because of the triangular inequality. The average dislocation density tensor can be written in terms of $\rho_{\mathrm{G}}$ and $\boldsymbol{l}$ as

$$
\boldsymbol{\alpha}=\rho_{\mathrm{G}} \boldsymbol{l} \otimes \boldsymbol{b} .
$$

In general, the average dislocation density tensor (16) does not fulfill the equation

$$
\partial_{t} \boldsymbol{\beta}^{p l}=-\boldsymbol{v} \times \boldsymbol{\alpha},
$$

if we interpret $\boldsymbol{v}$ as the pointwise vectorial average of the velocities along the dislocation lines.

By use of (10) together with averaging of (11), we can attempt to obtain an evolution equation for the averaged tensor. Writing this out, we obtain

$$
\partial_{t} \boldsymbol{\alpha}=-\operatorname{curl}\left\langle\sum_{\boldsymbol{c}} \delta_{\boldsymbol{c}} \boldsymbol{v} \times \frac{\mathrm{d} \boldsymbol{c}}{\mathrm{d} s} \otimes \boldsymbol{b}\right\rangle .
$$

The threefold product within the average can be written as a product of averages if, and only if, all dislocations within the averaging volume share the same tangent vector $\boldsymbol{l}=\mathrm{d} \boldsymbol{c} / \mathrm{d} s$ and velocity $\boldsymbol{v}$. This is possible either if only one dislocation is present (the discrete case) or if the dislocations form smooth line bundles. In these cases, $\rho=\rho_{\mathrm{G}}, \boldsymbol{\alpha}=\rho \boldsymbol{l} \otimes \boldsymbol{b}$, and (11) holds both on the local and on the averaged scale.

In the general case, however, the averaging volume contains dislocations of different orientations. Thus, averaging leads to a reduced dislocation density $\rho_{\mathrm{G}}<\rho$, and the dislocation density tensor does not obey (11) with $\boldsymbol{v}$ understood as the average velocity. Instead, additional terms appear in the evolution equation. The necessity of accounting for such terms, which have the formal structure of correlators between the dislocation density and velocity/line direction, has been recognized in the literature (see e.g. [17]). However, the derivation and closure of a higher-order theory which properly accounts for such terms requires a formidable theoretical effort. Until now, published attempts either remain at the level of declarations-of-intention, or provide phenomenological patches which do not really resolve the underlying theoretical problems. 


\section{Philosophical Magazine \& Philosophical Magazine Letters}

Philosophical Magazine

\subsection{Extended Continuum Theory}

To resolve the problem discussed above, the ECT distinguishes dislocation lines in a given spatial point $\boldsymbol{r}$ according to their line direction $\boldsymbol{l}$. As long as we are on the level of a discrete description, this is simply redundant. However, the physical rationale for this distinction becomes evident when we average over a mesoscopic volume: While it is in general unrealistic to assume that all dislocations contained in a mesoscopic volume have the same direction and move with the same velocity in response to an acting stress, it is much more realistic to assume that those dislocations which $d o$ have the same direction move in the same manner.

If dislocations move by glide only, their line direction can be parametrised by a scalar variable, for instance the angle $\varphi$ between line direction and Burgers vector. A point in configuration space is thus denoted by $(\boldsymbol{r}, \varphi)$. With regard to the numerical examples treated subsequently, we restrict ourselves to introducing ECT only for this situation. We note, however, that the theory is not restricted to this case but can be generalized to include out-of-plane dislocation motion and multiple slip systems (cf. [16]).

\subsubsection{Definition of the second order dislocation density tensor}

To define the dislocation density tensor and its averages in the three-dimensional configuration space we proceed in close analogy to the classical theory. Unlike in the classical case, however, we consider averages over so-called lifts of dislocation lines to the configuration space instead of averages over the spatial dislocation lines themselves. In order to introduce the concept of lifted curves we define a coordinate system such that the 1-direction points into the direction of the Burgers vector and the glide plane is spanned by the 1- and 2-directions. To each point $\boldsymbol{c}(s)=\left(c^{1}(s), c^{2}(s)\right)$ of a dislocation line we assign the angle $\varphi$ between the tangent $\mathrm{d} \boldsymbol{c} / \mathrm{d} s$ and the Burgers vector, that is $\varphi(s)=\arctan \left(\frac{\mathrm{d} c^{2}}{\mathrm{~d} s} / \frac{\mathrm{d} c^{1}}{\mathrm{~d} s}\right)$. We define the lift $\boldsymbol{C}$ of a given curve $\boldsymbol{c}$ to the three dimensional configuration space as

$$
\boldsymbol{C}(s)=\left(C^{1}(s), C^{2}(s), C^{3}(s)\right):=\left(c^{1}(s), c^{2}(s), \varphi(s)\right) .
$$

Figure 1 gives a visualisation of this concept. We note that the tangent to the lifted curve

$$
\frac{\mathrm{d} \boldsymbol{C}}{\mathrm{d} s}=\left(\frac{\mathrm{d} c^{1}}{\mathrm{~d} s}, \frac{\mathrm{d} c^{2}}{\mathrm{~d} s}, \frac{\mathrm{d} \varphi}{\mathrm{d} s}\right)=\left(\frac{\mathrm{d} \boldsymbol{c}}{\mathrm{d} s}, k(s)\right)
$$

contains as third coordinate the curvature $k(s)$ of the curve at $\boldsymbol{c}(s)$. As a consequence of this implicit definition of a metric the lifted curves $\boldsymbol{C}$ are not parametrised by arc length in the configuration space. ${ }^{1}$

By transferring the definition of $\delta_{c}$ to the lifted curve $C$ as

$$
\delta_{\boldsymbol{C}}(\boldsymbol{r})=\int_{\boldsymbol{C}} \delta(\boldsymbol{C}(s)-(\boldsymbol{r}, \varphi)) \mathrm{d} s,
$$

\footnotetext{
${ }^{1}$ It is worth noting that the definition of the second order dislocation density tensor does not necessarily require a metric or volume element. However, an invariant definition requires the use of advanced mathematical concepts as e.g. differential forms. We refrain from introducing these concepts in the current paper and refer the interested reader to [16] and especially [18] for a more thorough treatment.
} 
we define the dislocation density tensor of second order in complete analogy to the classical one as

$$
\boldsymbol{\alpha}_{(r, \varphi)}^{I I}=\left\langle\sum_{\boldsymbol{C}} \delta_{\boldsymbol{C}} \frac{\mathrm{d} \boldsymbol{C}}{\mathrm{d} s} \otimes \boldsymbol{b}\right\rangle .
$$

The sum is again taken over all dislocations in the system, and the averages are taken over a volume element in configuration space which is centered at $\boldsymbol{r}$ and $\varphi$.

It is illustrative to express also $\boldsymbol{\alpha}^{I I}$ as the product of a density function $\rho_{(r, \varphi)}$ and a generalised tangent which we denote by $\boldsymbol{L}_{(r, \varphi)}$. The definition of these two objects is not completely analoguous to the classical ones. The density function is defined as

$$
\rho_{(r, \varphi)}=\left\|\left\langle\sum_{\boldsymbol{C}} \delta_{\boldsymbol{C}} \frac{\mathrm{d} \boldsymbol{C}}{\mathrm{d} s}\right\rangle\right\|_{r},
$$

where $\|\cdot\|_{r}$ measures the length of the spatial projection of a vector in the configuration space. The tangents to the lifted curves have the property

$$
\left\|\frac{\mathrm{d} \boldsymbol{C}}{\mathrm{d} s}\right\|_{r}=\left\|\frac{\mathrm{d} \boldsymbol{c}}{\mathrm{d} s}\right\|=1
$$

In (23), the averaging volume - a volume element in configuration space - contains dislocations of one orientation only. Therefore, there is no cancellation - e.g. of dislocations of opposite directions - during averaging of the spatial tangent vectors. Therefore, the norm $\|\cdot\|_{r}$ may be interchanged with the averaging. We thus find that $\rho_{(r, \varphi)}$ gives the spatial line length per volume (of the configuration space) of dislocations at $\boldsymbol{r}$ with direction $\boldsymbol{l}(\varphi)=(\cos \varphi, \sin \varphi)$. The generalised tangent is defined as

$$
\boldsymbol{L}_{(r, \varphi)}=\frac{\left\langle\sum_{\boldsymbol{C}} \delta_{\boldsymbol{C}} \frac{\mathrm{d} \boldsymbol{C}}{\mathrm{d} s}\right\rangle}{\rho_{(r, \varphi)}}
$$

We note that the first two components of $\boldsymbol{L}_{(r, \varphi)}$ are just the canonical spatial direction at $\varphi$ and the third component contains the average curvature $k_{(r, \varphi)}$, that is

$$
\boldsymbol{L}_{(r, \varphi)}=\left(\cos \varphi, \sin \varphi, k_{(r, \varphi)}\right)=\left(\boldsymbol{l}(\varphi), k_{(r, \varphi)}\right)
$$

From the definitions (22), (23) and (25) we easily find

$$
\boldsymbol{\alpha}_{(r, \varphi)}^{I I}=\rho_{(r, \varphi)} \boldsymbol{L}_{(r, \varphi)} \otimes \boldsymbol{b}
$$

The classical dislcoation density tensor $\alpha$ can be evaluated from the density function $\rho_{(r, \varphi)}$ as

$$
\boldsymbol{\alpha}_{(r, \varphi)}=\int_{0}^{2 \pi} \rho_{(r, \varphi)} \boldsymbol{l}_{\varphi} \mathrm{d} \varphi \otimes \boldsymbol{b}
$$




\section{Page 9 of 30}

4

5

7

9

10

$$
\begin{aligned}
\boldsymbol{\alpha}_{(r, \varphi)}^{I I} & =\rho_{(r, \varphi)}\left(\begin{array}{ll}
b^{1} \cos \varphi & b^{2} \cos \varphi \\
b^{1} \sin \varphi & b^{2} \sin \varphi \\
b^{1} k_{(r, \varphi)} & b^{2} k_{(r, \varphi)}
\end{array}\right) \\
& =\rho_{(r, \varphi)}\left(\begin{array}{ll}
\cos \varphi & 0 \\
\sin \varphi & 0 \\
k_{(r, \varphi)} & 0
\end{array}\right) b,
\end{aligned}
$$

where the latter follows from the chosen coordinate system for which we took the 1-direction parallel to the Burgers vector and hence find $\boldsymbol{b}=(b, 0)$.

We remark that in analogy to (7) also the generalised divergence of $\boldsymbol{\alpha}^{I I}$ must vanish (cf. [16]), which likewise reflects the physical fact that dislocation lines do not start or end inside a crystal. With (29) this condition reads

$$
\cos \varphi \partial_{x} \rho+\sin \varphi \partial_{y} \rho+\partial_{\varphi}(\rho k)=0
$$

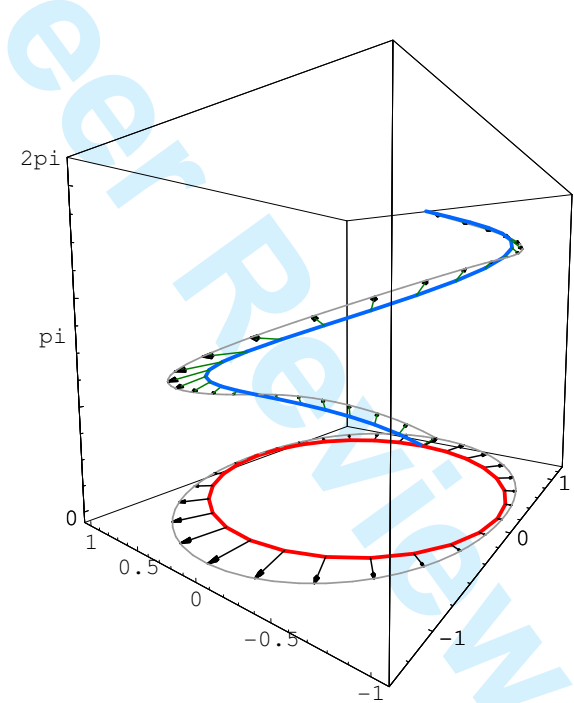

Figure 1. Continuous lifted curve in the configuration space. The lower, closed loop is the spatial loop, the upper line is the lifted loop with additional orientation information. The arrows attached to the lower loop indicate the spatial velocity, the arrows attached to the upper curver indicate the generalised velocity along the line. The rotational velocity $\vartheta$ is the vertical component of the generalised velocity.

\subsubsection{The generalised velocity}

In the preceding section we defined the dislocation density tensor $\boldsymbol{\alpha}^{I I}$ as an average of density functions characterising discrete lifted curves. In order to obtain the evolution of $\boldsymbol{\alpha}^{I I}$, we need to consider the velocity of the lifted curve in the configuration space. As may be seen in Figure 1 the velocity of the lifted curve contains, besides the spatial (horizontal) velocity $\boldsymbol{v}$ orthogonal to the spatial dislocation line, also a component in the $\varphi$ direction which accounts for the rotation of line segments during dislocation motion.

It is straightforward to show that the pseudo-scalar rotation velocity $\vartheta$ of a moving curve parametrised by arc length $s$ is determined by the change of velocity along 
the line. For the pseudo-scalars $v, k$ and $\vartheta$ we use the following sign convention: We consider the pseudo-scalar velocity of a positively oriented loop as positive if the loop expands (compare Fig. 2). The curvature of such a loop is also considered as positive. From this sign convention it follows that the pseudo-scalar rotational velocity is

$$
\vartheta(s)=-\frac{\mathrm{d} v(s)}{\mathrm{d} s}
$$

Using these conventions, we define the generalised velocity at a point $\boldsymbol{C}(s)$ of a lifted discrete curve as

$$
\boldsymbol{V}^{d}(s)=(v \sin \varphi(s),-v \cos \varphi(s), \vartheta(s)) .
$$

We note that unlike the spatial velocity, which is orthogonal to the dislocation line, the generalised velocity is in general not orthogonal to the lifted curve.

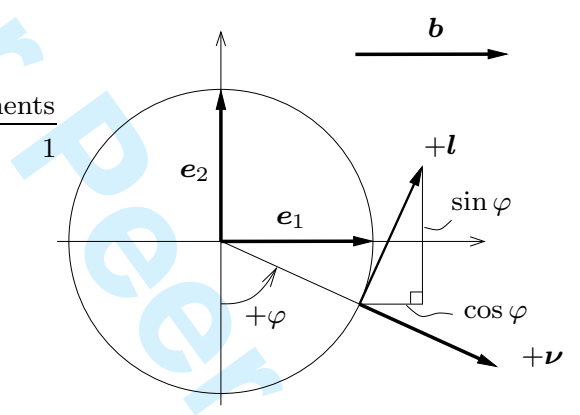

Figure 2. Sign convention for the line orientation $\varphi$, tangent $\boldsymbol{l}$ and normal $\boldsymbol{\nu}$ on the parameterized unit circle

\section{The evolution of $\boldsymbol{\alpha}^{I I}$}

In analogy with the classical definition we define the generalised (discrete) dislocation current

$$
\boldsymbol{J}^{I I, d}=\sum_{C} \boldsymbol{V}^{d} \times \delta_{C} \frac{d \boldsymbol{C}}{\mathrm{d} s} \otimes \boldsymbol{b} .
$$

The evolution of the averaged dislocation density tensor of second order is obtained in analogy to (18):

$$
\partial_{t} \boldsymbol{\alpha}_{(r, \varphi)}^{I I}=-\operatorname{curl}\left\langle\boldsymbol{J}^{\mathrm{II}, \mathrm{d}}\right\rangle_{(r, \varphi)} .
$$

The curl operator again acts on the columns of the coefficient matrix of a tensor. The difference to the evolution of the classical dislocation density tensor is that in (34) the product appearing in the generalised dislocation current (compare (33)) can be understood as a product of averages under much weaker assumptions on the dislocation configuration. The most important assumption in this respect is that nearby dislocations of the same line direction have the same curvature, which is reasonable in quasi-static situations, where the curvature essentially balances the local stress. Under this assumption, the average dislocation current can be written as

$$
\boldsymbol{J}^{I I}=\boldsymbol{V}_{(r, \varphi)} \times \boldsymbol{\alpha}_{(r, \varphi)}^{I I},
$$




\section{Page 11 of 30 \\ Philosophical Magazine \& Philosophical Magazine Letters}

where the average velocity $\boldsymbol{V}_{(r, \varphi)}=\left\langle\boldsymbol{V}^{d}\right\rangle$ can be expressed in terms of the average pseudo-scalar velocity $v_{(r, \varphi)}=\langle v\rangle$ and rotation velocity $\vartheta_{(r, \varphi)}=\langle\vartheta\rangle$ as

$$
\boldsymbol{V}_{(r, \varphi)}=\left(v_{(r, \varphi)} \sin \varphi,-v_{(r, \varphi)} \cos \varphi, \vartheta_{(r, \varphi)}\right)
$$

Consequently, the evolution equation for the dislocation density tensor of second order is obtained as

$$
\partial_{t} \boldsymbol{\alpha}_{(r, \varphi)}^{I I}=-\operatorname{curl}\left(\boldsymbol{V}_{(r, \varphi)} \times \boldsymbol{\alpha}_{(r, \varphi)}^{I I}\right) .
$$

\subsubsection{Evolution equations for the scalar field variables}

As we saw in (29), in the single glide situation under consideration $\boldsymbol{\alpha}^{I I}$ is completely defined by the two scalar fields $\rho_{(r, \varphi)}$ and $k_{(r, \varphi)}$. Hence, the evolution equation for $\boldsymbol{\alpha}^{I I}$ can be translated into evolution equations for these functions. As the derivation of these evolution equations involves either abstract methods or simple but lengthy calculations we only give the result and refer the reader to [16] for details:

$$
\begin{aligned}
& \partial_{t} \rho=-\left(\operatorname{div}(\rho \boldsymbol{v})+\partial_{\varphi}(\rho \vartheta)\right)+\rho v k, \\
& \partial_{t} k=-v k^{2}-\nabla_{\boldsymbol{L}}^{2}(\boldsymbol{v})-\nabla_{\boldsymbol{V}}(k) .
\end{aligned}
$$

Here, $\nabla_{\boldsymbol{L}}^{2}$ and $\nabla_{\boldsymbol{V}}$ denote the second derivative along the generalised line direction and the first derivative along the generalised velocity direction, respectively. To avoid cumbersome notation we dropped the index ${ }_{(r, \varphi)}$. In the following if no indices occur we always will imply that all quantities are evaluated at the point $(\boldsymbol{r}, \varphi)$ in the configuration space $\mathbb{R} \times \mathbb{R} \times[0,2 \pi)$.

\subsubsection{Evolution equation for the plastic distortion}

The evolution equation for the plastic distortion $\boldsymbol{\beta}^{p l}$ takes a similar form as (10) in the classical formulation:

$$
\partial_{t} \boldsymbol{\beta}^{p l}=\int_{0}^{2 \pi} \rho_{(r, \varphi)} v_{(r, \varphi)} \mathrm{d} \varphi \boldsymbol{n} \otimes \boldsymbol{b} .
$$

This quantity provides the link to the material response on the continuum level as introduced in section 2.1.

\section{Numerical Implementation}

\subsection{Geometry}

As in the preceding section we assume that all dislocations are moving on a single glide system with glide plane normal $\boldsymbol{n}=\boldsymbol{e}_{3}$ and Burgers vector $\boldsymbol{b} \equiv \boldsymbol{b}_{1}$. The system is assumed homogeneous in the $\boldsymbol{e}_{3}$ direction, i.e., we consider a statistically homogeneous assembly of active glide planes with constant average spacing. 


\subsection{Discretization of the problem}

We define a discrete time step $\Delta t:=t^{i+1}-t^{i}$ with $i \in \mathbb{N}^{+}$. A forward Euler scheme is used for numerical time integration of the evolution equations. Hence, only values at step $t^{i}$ are needed to compute the new values at step $t^{i+1}$; no additional history variables need to be stored. This is beneficial for the required computer memory, which is an issue as the multidimensional structure of the configuration space requires a large amount of information to be stored for multiple fields. Furthermore, we use a fixed mesh which demands a relatively fine space discretisation with corresponding large storage requirements.

The forward Euler time integration scheme is an unstable scheme which is only first order accurate and suffers from dispersion. Our main reason for using this scheme is that its simple explicit structure allows for an easy identification of the separate effects stemming from the different terms in the evolution equations. Implicit time integration schemes, on the other hand, require additional linearization steps along with the iterative solution of the resulting difference equations. It is then not straightforward to distinguish effects stemming e.g. from the discretisation, the initial values, the approximative nature of the Newton linearisation or the partial differential equations themselves. For the explicit Euler scheme used together with a compatible choice of numerical derivatives, we could identify a 'working regime' within which this integration method was numerically reliable and could be used to 'test' more fundamental aspects of the numerical implementation ${ }^{1}$.

The 3-dimensional configuration space is discretised by a uniform mesh. It turned out that a sufficient resolution in the angular direction requires between 60 and 120 nodes. In the case of quasi-discrete lines we use a discrete Gaussian distribution to approximate the Dirac delta function. Since we use a fine spatial resolution for this representation (e.g. about 60 nodes to discretise the part of the Gauss function which is $>0.1 \%$ of the peak value) dispersive effects can be neglected.

Derivatives w.r.t. the configuration space are approximated by finite differences. Derivatives which govern transport were approximated by an upwind scheme. This is a numerical scheme which uses information about the flow direction (e.g. the direction of propagation of a wave) to determine whether to use a forward or backward difference stencil, such that only information from upstream of the flow is used. Similar schemes are widely used to solve hyperbolic partial differential equations in computational fluid dynamics and reduce oscillations in direction of wave travel (e.g. [19]).

The upwind method based on first order forward and backward differences is oscillation-free but at the cost of a very strong 'smearing-out' effect in direction of wave travel caused by a large amount of 'numerical viscosity'. We therefore use a scheme based upon second order accurate forward and backward differences, which is of much better quality than the first order scheme but slightly oscillatory [20]. As a very simple remedy against the undershoot effect causing the oscillations, we cut off the (unphysical) negative density values and distribute this negative amount over all nodes with positive density. This procedure causes a steepening gradient on the downstream side of the wavefront and a slight increase of the wave's maximum - both of which are only minor effects in the cases we study.

All derivatives for nonconvective (e.g. diffusion) terms were approximated by central difference schemes of second order accuracy.

\footnotetext{
${ }^{1}$ In our case, we pragmatically consider a numerical scheme to be well-behaving within the 'working regime' if a positive half-wave can be propagated for more than 10000 steps with a velocity $v=1.0$, the mesh width $h=1.0$ and a time step $\Delta t=0.1$, such that the peak of the wave can clearly be identified and no oscillations occur.
} 


\section{Page 13 of 30} $[22]$

To determine the time step size we use the Courant-Friedrichs-Lewy condition

$$
\left|v \frac{\Delta t}{\Delta x}\right| \leq 1
$$

which serves as a (necessary) stability criterion for purely advective equations of the form

$$
\partial_{t} \rho+v \partial_{x} \rho=0
$$

that are solved by explicit time integration schemes such as the forward Euler scheme. In our simulations we accordingly choose $\Delta t \approx 0.1 \Delta x / \hat{v}$ where $\hat{v}$ is the largest occurring velocity.

\section{Test Cases}

In this section we investigate two examples, both of which are simple enough such that we still can easily predict the outcome while exploring the evolution equations of ECT. The first example treats a homogeneous distribution of circular loops. This case, which can be solved analytically, illustrates some basic properties of the theory. The second example treats a single quasi-discrete circular dislocation loop. This serves to illustrate and address issues related to the numerical discretisation of the system in a higher-dimensional configuration space.

\subsection{Homogeneous distribution of expanding loops}

For a homogeneous distribution of equidistant loops with the same initial radius $r_{0}$ in an unbounded glide plane, all derivatives of $\rho, k$ and $v$ w.r.t. the configuration space vanish. Then the system of evolution equations (38) and (39) reduces to

$$
\begin{aligned}
\partial_{t} \rho & =\rho v k, \\
\partial_{t} k & =-v k^{2} .
\end{aligned}
$$

(43) only consists of a production term, which yields the change of line length during expansion of the loops, while (44) governs the change of curvature $k(t)=1 / r(t)$ due to the expansion. Note that here $\rho$ and $v$ are independent of $\varphi$. Thus, we can directly obtain the evolution of the plastic distortion from (40) as

$$
\partial_{t} \beta^{p l}=2 \pi \rho b v .
$$

We could have obtained the same evolution equation for the curvature by considering the change of radius of a loop with initial radius $r_{0}$ which expands with 
the velocity $v$ :

$$
\begin{gathered}
k=\frac{1}{r(t)}=\left(\frac{1}{k_{0}}+v t\right)^{-1} \\
\Rightarrow \partial_{t} k=-v\left(\frac{1}{k_{0}}+v t\right)^{-2}=-v k^{2} .
\end{gathered}
$$

The evolution equation for the scalar density can then be obtained by considering homogeneously distributed loops with mean center-to-center spacing $d$ and initial radius $r_{0}$. The density is the line length $2 \pi r(t)$ per volume, i.e. in terms of $k$

$$
\rho=\frac{2 \pi}{k} \frac{1}{d^{3}}
$$

We obtain the evolution equation as time derivative of (48):

$$
\partial_{t} \rho=\frac{2 \pi}{d^{3}} \partial_{t}\left(\frac{1}{k}\right)=-\rho \frac{\partial_{t} k}{k}=\rho v k
$$

Hence, the case of a homogeneous distribution of equidistant, expanding or shrinking loops with the same initial radius is correctly represented by the theory.

In our introduction we mentioned that an essential shortcoming of Kosevich's and El-Azab's continuum theories is manifested by the fact that the kinematic behavior of a distribution of straight dislocation lines is not distinguished from that of a distribution of loops with the same radii. The ECT, however, reproduces the correct increase of line length for the evolving system of distributed loops, while for the case of the straight line distribution the theory yields pure advection without change in line length.

\subsection{Quasi-discrete expanding circular loop}

We again assume constant velocity and curvature such that there is no rotational velocity and no change of curvature along the line. As all derivatives of $v$ and $k$ are zero the evolution equations now simplify to

$$
\begin{aligned}
& \partial_{t} \rho=-\operatorname{div}(\rho \boldsymbol{v})+\rho v k, \\
& \partial_{t} k=-v k^{2} .
\end{aligned}
$$

The first part of the evolution equation for $\rho$ governs spatial transport, whereas the second part accounts for changes in line length due to expansion or shrinkage of curved dislocation segments (here forming circular loops). The change of curvature which goes along with the expansion or shrinkage of segments is determined by the evolution equation for $k$.

\subsubsection{Numerical results: The problem of line fragmentation}

As an approximation to a discrete dislocation loop we generate a continuous density function by replacing the $\delta$-distribution in (21) with a Gaussian distribution. The spatial projection of the initial distribution, that is the total dislocation density, is depicted in Fig. 3(a). Time integration of the evolution equations (50) and (51) leads to a spatial density distribution as shown in Fig. 3(b). The growth of 


\section{Page 15 of 30 \\ Philosophical Magazine \& Philosophical Magazine Letters}

1

4

7

9

10

11

12

13

14

15

16

17

18

19

20

21

22

23

24

25

26

27

28

29

30

31

32

33

34

35

36

37

38

39

40

41

42

43

44

45

46

47

48

49

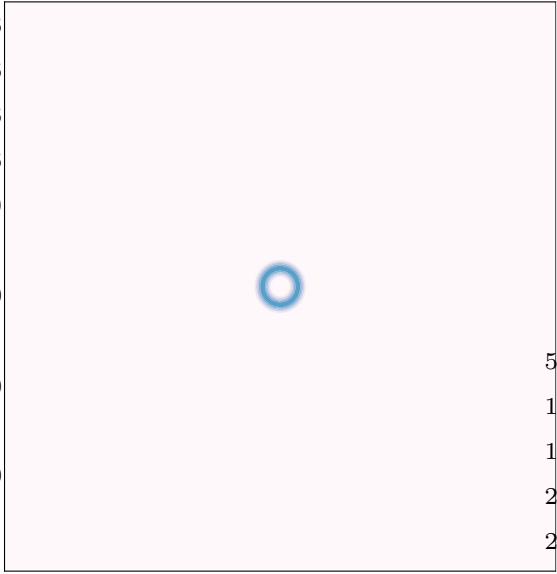

(a) Initial configuration $(R=30$ nodes $)$

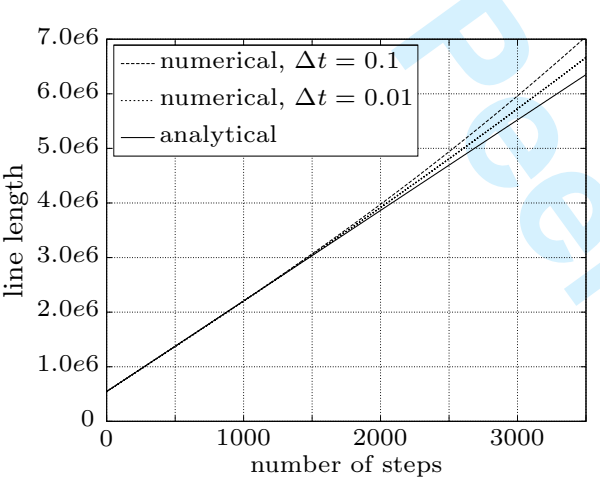

(c) Temporal evolution of the systems' total line length

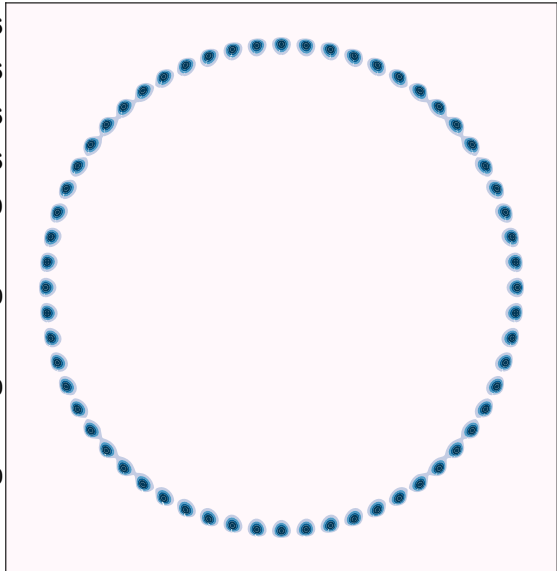

(b) Expanded loop after 3500 steps with $\Delta t=0.1$ and $v=1.0(R=3800$ nodes)

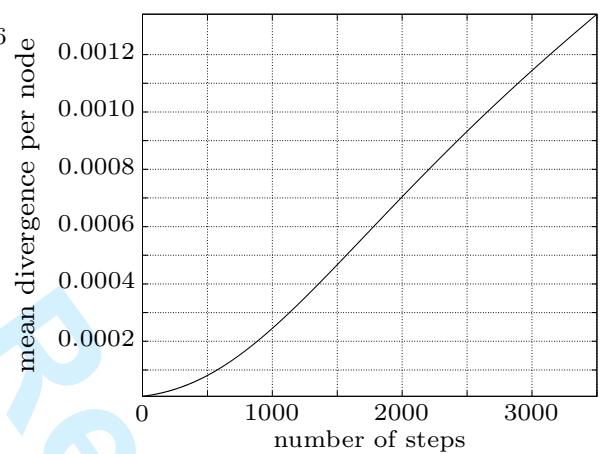

(d) Temporal evolution of the mean divergence per node, $\left(1 / N_{\text {nodes }}\right) \sum \|$ div $\boldsymbol{\alpha}^{I I} \|$

Figure 3. Evolution of the (spatial) total dislocation density for an expanding loop as obtained from solving Equation (50) and (51): (a) initial configuration, (b) end configuration. The loop was discretized with 60 nodes in $\varphi$-direction. While the total amount of density integrated over the plane is - up to a numerical error - predicted correctly (c), the loop heavily fragments. This also displays in the evolution of the numerical divergence depicted in (d).

the total dislocation line length, as expressed by the volume integral of the density function over the configuration space, is described correctly (Fig. 3(c)), except for a small deviation which is caused by discretisation errors. Nonetheless, the density distribution gets spatially fragmented as the loop expands. This fragmentation is directly related to a growing divergence of $\boldsymbol{\alpha}^{I I}$. As the time derivative of $\boldsymbol{\alpha}^{I I}$ is a curl (compare (37)) the evolution equations themselves conserve the solenoidality of $\boldsymbol{\alpha}^{I I}$. However, finite difference schemes in general can not guarantee that the discrete curl - and div - operators fulfill the relation div curl $=0$. This shortcoming manifests itself in a strong increase of the mean divergence $\left(1 / N_{\text {nodes }}\right) \sum\left\|\operatorname{div} \boldsymbol{\alpha}^{I I}\right\|$ as shown in Fig. 3(d). This increase mirrors the visible fragmentation of the dislocation density distribution during expansion of the loop. This fragmentation is unphysical. Moreover, in the case of inhomogeneous velocity fields the temporal evolution depends on derivatives of the density along the generalised line direction. The numerical evaluation of these derivatives becomes completely inaccurate if loop fragmentation becomes pronounced. 
We note that other authors have observed similar problems, see e.g. [21] where the problem is addressed by utilising polar coordinates. This avoids fragmentation, but the approach can be used only for the special case of loops with the coordinate origin as their center, and not for the evolution of general distributions. The remainder of this section deals with generic strategies that avoid fragmentation within the framework of the chosen finite difference scheme.

\subsubsection{Direct relaxation of the dislocation density tensor $\boldsymbol{\alpha}^{I I}$}

A straightforward method to maintain solenoidality of the density distribution is to minimize the divergence of $\boldsymbol{\alpha}^{I I}$ by means of a sequence of 'relaxation' steps following each time step. This process ensures iteratively that div $\boldsymbol{\alpha}^{I I}$ as a measure of fragmentation is kept below a given tolerance. We minimise the total divergence of $\boldsymbol{\alpha}^{I I}$ using the iteration formula

$$
\boldsymbol{\alpha}_{\text {new }}^{I I} \Leftarrow \boldsymbol{\alpha}_{\text {old }}^{I I}+\lambda \operatorname{grad} \operatorname{div}\left(\boldsymbol{\alpha}^{I I}\right),
$$

where $\lambda$ is a factor controlling the step size, which can be adjusted to achieve efficient relaxation while avoiding 'overshoots'. However, this method has clear drawbacks: The operators grad div (.) introduce second derivatives which have a diffusive effect and suppress fragmentation by homogenizing the dislocation density distribution along the dislocation line. However, the diffusive effect also acts in the perpendicular direction and causes a broadening of the line. Furthermore, the iteration scheme (52) conserves $\boldsymbol{\alpha}^{I I}$ and not $\rho$, causing unphysical changes in dislocation density during the relaxation step. An improved relaxation scheme which conserves the total density and avoids diffusive 'flattening' of the dislocation density distribution is obtained by modifying (52) such that $\rho$ is relaxed only along the line:

$$
\rho_{\text {new }} \Leftarrow \rho_{\text {old }}+\lambda \nabla_{\boldsymbol{l}}^{2} \rho,
$$

where the relaxation is again carried out to minimize $\sum\left\|\operatorname{div} \boldsymbol{\alpha}^{I I}\right\|$.

Irrespective of the method used, relaxation of the system is computationally expensive. Achieving a well-relaxed configuration requires multiple relaxation steps, each of which is computationally about as expensive as one time integration step for the evolution equations. To obtain a computationally more efficient method for preserving solenoidality we take a closer look at the mechanism that leads to the fragmentation and devise a remedy which restores the unfragmented state without introducing an additional iterative process.

\subsubsection{Correction of line fragmentation by tangential diffusion}

To better understand loop fragmentation from a geometrical point of view, we consider a curved dislocation line in the configuration space (for simplicity we envisage a circular loop but the argument extends to any curved line). To obtain a continuous and differentiable density distribution we need to approximate the Dirac $\delta$-function representing the discrete line in terms of continuous and differentiable functions. Doing this on a discrete grid amounts to a discrete convolution with an approximation function, i.e., we map (a subset of) $\mathbb{R}$ to (a subset of) $\mathbb{N}$. For poor resolution in $\varphi$-direction and a better resolution in $x$ and $y$ direction, Fig. 4 shows the density distribution obtained with Gaussian functions. It is important to realize that due to the mapping, each of the 'blobs' representing segments of the dislocation line is located on a particular $\varphi$-plane. As all points of the blob move into the same direction $\boldsymbol{v}$, which is perpendicular to the spatial line direction $\boldsymbol{l}(\varphi)$, the blobs are drifting apart during loop expansion and the line becomes fragmented. 


\section{Page 17 of 30}

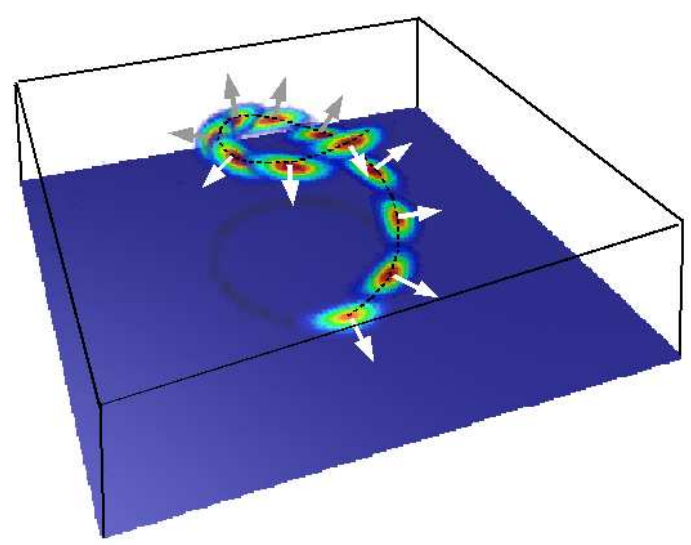

Figure 4. Discrete density distribution which corresponds to a lifted line for a bad resolution in $\varphi$-direction (12 nodes only) chosen deliberately for demonstration purposes. Each of these blobs is moving as a whole along the direction indicated by the arrows.

We consider the effect of loop expansion at two successive time steps $t^{i}$ and $t^{i+1}$ and its impact on the change of arc length of a line segment $s^{i}$ for the case of a continuous lifted loop and for a lifted loop with discretized orientations. Fig. 5 shows the geometrical relations between these two cases. To understand how the differences affect dislocation density evolution, we may then think of a single lifted line as as a bundle of parallel lines with partial Burgers vectors and characterize the bundle by a space-dependent density $\rho$.

Let us first consider the expansion of a continuous lifted loop. As the loop expands in the spatial plane a circle segment $s^{i}$ with cone angle $\Delta \varphi$ gets elongated to $s^{i+1}$ (cf. Fig. 5(a)). Expressing this relation in terms of the curvature we get

$$
k^{i}=\frac{\Delta \varphi}{s^{i}} \quad \text { and } \quad k^{i+1}=\frac{\Delta \varphi}{s^{i+1}} .
$$

The increment of spatial arc length $\Delta s$ from step $i$ to step $i+1$ then is defined through

$$
\Delta s:=s^{i+1}-s^{i}=\Delta \varphi\left(\frac{1}{k^{i+1}}-\frac{1}{k^{i}}\right)
$$

The arc length increase stems from the fact that the line orientation along the circle segment varies between $\varphi_{0}-\frac{\Delta \varphi}{2}$ and $\varphi_{0}+\frac{\Delta \varphi}{2}$, cf. Fig. 5(a), leading to divergent trajectories and a separation of the endpoints of the segment. For a bundle of lines, the local density within the bundle remains unchanged while the overall density increases as the bundle occupies more space.

The numerically discretised loop, on the other hand, consists of 'straight' line segments (the orientation is constant within each segment). Hence, each point of the segment translates into the same direction and no increase in line length $\Delta s$ can take place (thick black lines in Fig. 5(b)). However, ECT is based on the description of continuous lifted lines and therefore its equations are constructed to generate the correct additional amount of density (which is governed by the production term $\rho v k$ of (38)). Unfortunately, after discretization the transport terms fail to locate this amount in the right place.

The consequences are illustrated in Fig. 6: In case of a bundle of continuous lifted lines, Fig. 6 (a), additional line length is introduced by stretching the curved segments while the density remains constant. In case of a bundle with discrete orientations, Fig. 6(b), on the other hand, the segment length remains constant, 


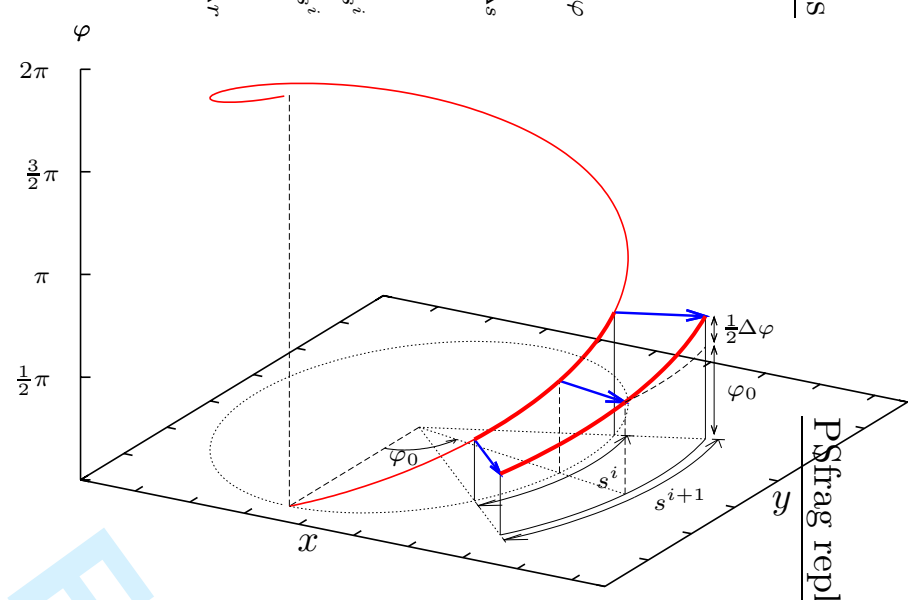

(a) line segment during ideal mathematical expansion of a lifted loop

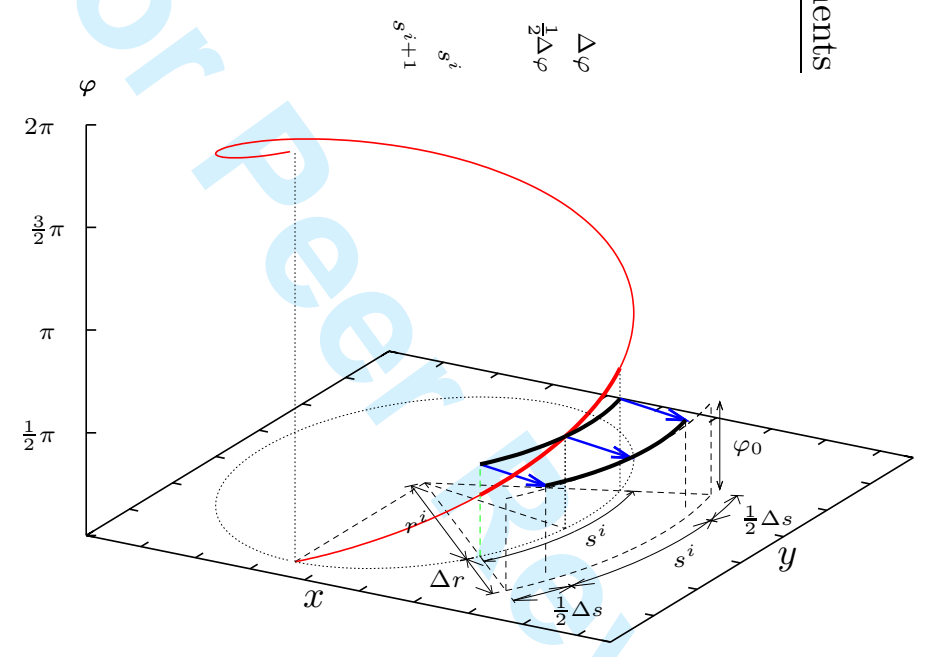

(b) line segment during numerically approximated lifted loop expansion

Figure 5. Expansion of a continuous loop and the effect due to numerical discretisation: A lifted loop with radius $r^{i}$ expands by $\Delta r$ affecting the lines' inclination (the curvature) and its length. In the case of the continuouos loop expansion the line segment gets rotated (by reduction of curvature) and stretched. The numerical scheme takes care of the rotation by an evolving curvature (not shown) but otherwise can only translate the segment without stretching.

leading to fragmentation, while the additional line length causes an increase in density,

$$
\Delta \rho=\left(\frac{s^{i+1}}{s^{i}}-1\right) \rho=\frac{\Delta s}{s^{i}} \rho .
$$

This suggests to remedy the situation by redistributing the additional density along the line.

The intuitively obvious method to do this is shown in Fig. 6(c): We might remove the added density $\Delta \rho$ and distribute it to the left and right of the original segments, such as to extend these segments by exactly the amount needed to restore connectivity. Unfortunately, numerical implementation of this idea is not straightforward since we are working with a fixed spatial grid where the notation of 'segment length' is not defined. Instead, we implement a diffusive spreading scheme: We 


\section{Page 19 of 30}

8

9

10

11

12

13

14

15

16

17

18

19

20

21

22

23

24

25

26

27

28

29

30

31

32

33

34

35

36

37

38

39

40

41

42

43

44

45

46

47

48

49

50

51

52

53

54

55

56

57

58

59

60

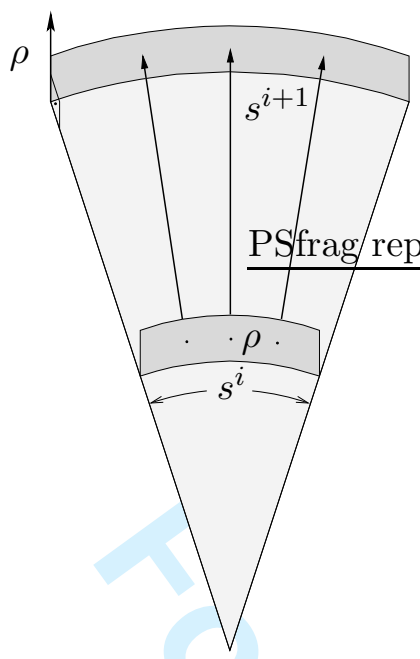

(a) line segment bundle during expansion of a loop with continuous orientation

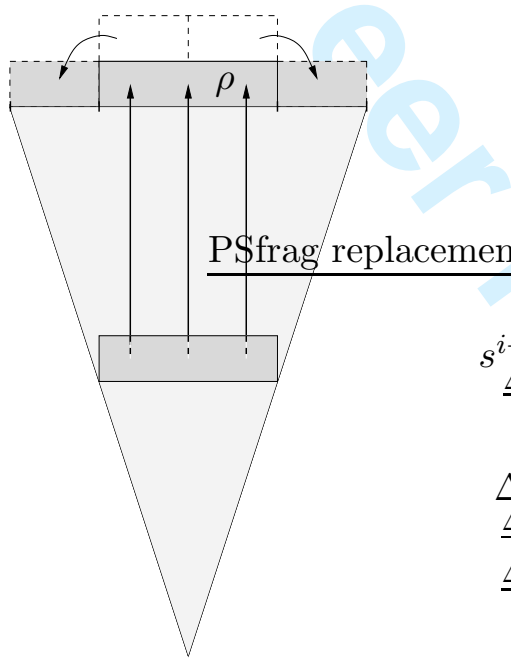

(c) density redistribution to 'stretch' the segments to the correct length

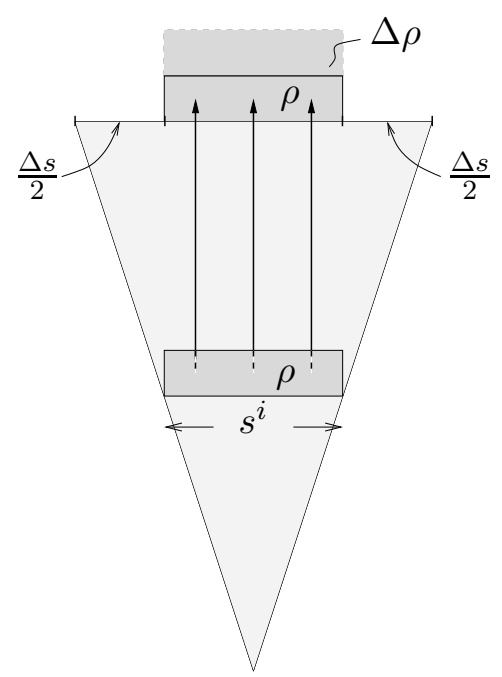

(b) line segment bundle during expansion of a loop with discretized orientation

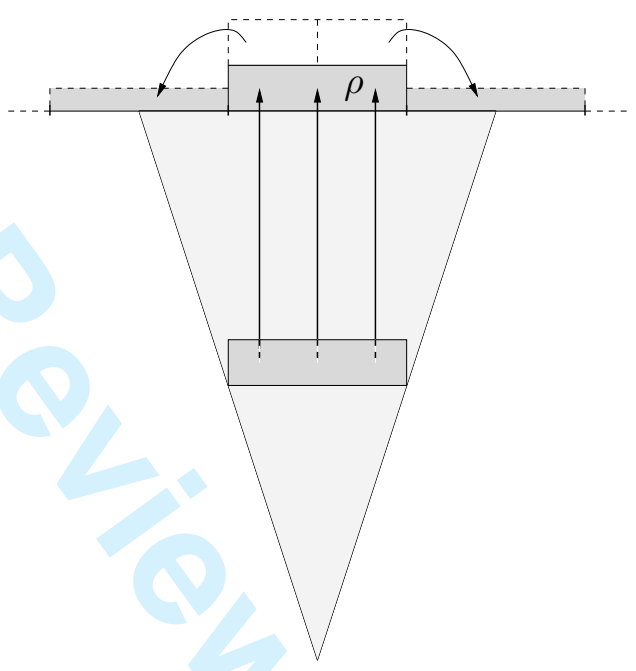

(d) diffusive density redistribution

Figure 6. Diagrams illustrating schematically the density evolution of a bundle of expanding dislocation loops. Graph (a) represents loops with continuous segment orientation. The other graphs refer to loops with discretized orientation: Graph (b) shows the evolution before correction, (c) after restoring line continuity by density re-distribution, and (d) with diffusive density re-distribution.

continue each segment along its direction into a sequence of segments of similar orientation and length as shown in Fig. 6(d), and distribute the excess density on its two nearest neighbors in this sequence. Conversely, if these neighbors already carry density (and thus produce additional density $\Delta \rho$ during loop expansion) half of the newly generated density from both neighbors is distributed back onto the original segment.

Thus, at each time step the density at $\boldsymbol{r}$ is diminished by the newly created density $\Delta \rho(\boldsymbol{r})$ but increased by the contributions $\Delta \rho_{\mathrm{L}}(\boldsymbol{r})$ and $\Delta \rho_{\mathrm{R}}(\boldsymbol{r})$ from the 
neighbors at $\boldsymbol{r} \pm s^{i} \boldsymbol{l}$,

$$
\begin{aligned}
& \Delta \rho_{\mathrm{L}}(\boldsymbol{r})=\frac{\Delta s}{2 s^{i}} \rho\left(\boldsymbol{r}-s^{i} \boldsymbol{l}\right), \\
& \Delta \rho_{\mathrm{R}}(\boldsymbol{r})=\frac{\Delta s}{2 s^{i}} \rho\left(\boldsymbol{r}+s^{i} \boldsymbol{l}\right) .
\end{aligned}
$$

The total density change due to this diffusive re-distribution is then given by

$$
\Delta \rho^{\operatorname{diff}}(\boldsymbol{r})=\frac{\Delta s}{2 s^{i}}\left[\rho\left(\boldsymbol{r}-s^{i} \boldsymbol{l}\right)+\rho\left(\boldsymbol{r}+s^{i} \boldsymbol{l}\right)-2 \rho(\boldsymbol{r})\right] .
$$

Taylor expansion around $\boldsymbol{r}$ of first two density terms on the right-hand side yields

$$
\begin{aligned}
& \rho\left(\boldsymbol{r}-s^{i} \boldsymbol{l}\right) \approx \rho(\boldsymbol{r})-s^{i} \nabla_{\boldsymbol{l}} \rho(\boldsymbol{r})+\frac{\left(s^{i}\right)^{2}}{2} \nabla_{\boldsymbol{l}}^{2} \rho(\boldsymbol{r}) \\
& \rho\left(\boldsymbol{r}+s^{i} \boldsymbol{l}\right) \approx \rho(\boldsymbol{r})+s^{i} \nabla_{\boldsymbol{l}} \rho(\boldsymbol{r})+\frac{\left(s^{i}\right)^{2}}{2} \nabla_{\boldsymbol{l}}^{2} \rho(\boldsymbol{r}),
\end{aligned}
$$

where $\nabla_{l}$ and $\nabla_{l}^{2}$ are the first and second derivatives along the spatial direction of the line. Inserting the above two equations into (59) yields

$$
\partial_{t} \rho^{\mathrm{diff}}(\boldsymbol{r})=\lim _{\Delta t \rightarrow 0}\left(\frac{\Delta s}{\Delta t} \frac{1}{2} s^{i} \nabla_{\boldsymbol{l}}^{2} \rho(\boldsymbol{r})\right)
$$

which is the general formulation of the diffusive correction term.

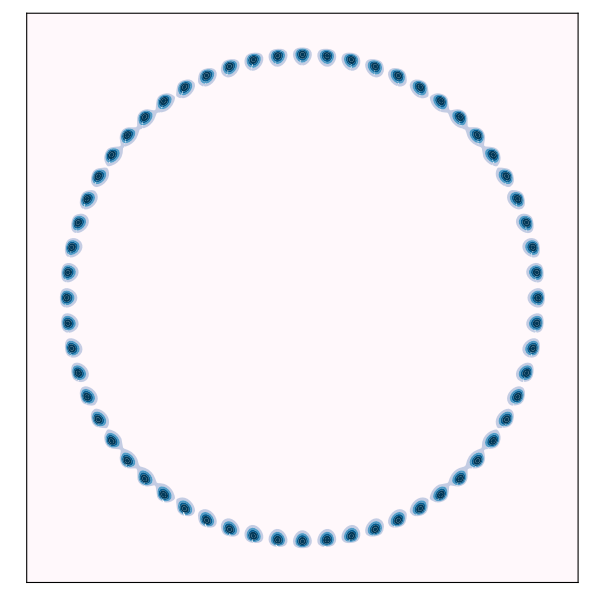

(a) Expanded loop without diffusive cor-

rection term after 3500 steps with $\Delta t=0.1$ and $v=1.0(R=380$ nodes $)$

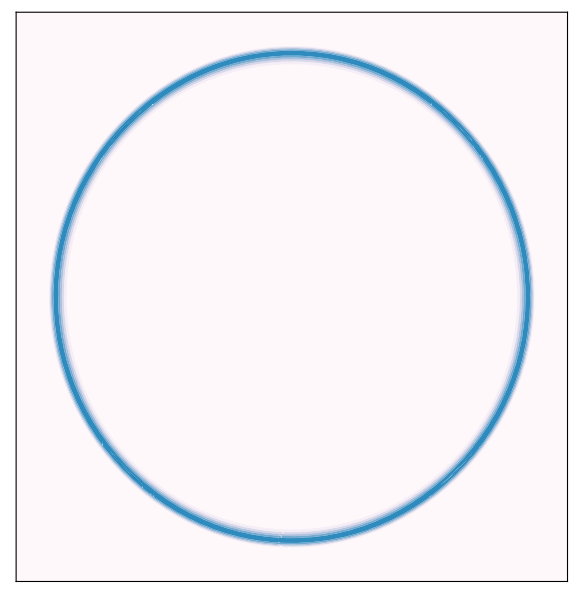

(b) Expanded loop with diffusive correction term after 3500 steps with $\Delta t=0.1$ and $v=1.0(R=380$ nodes $)$

Figure 7. Expansion of a circular loop with 60 nodes in $\varphi$-direction: projection of the density $\rho_{(r, \varphi)}$ into the spatial plane. The initial configuration was the same as in Fig. 3(a).

As fragmentation is related to orientation space discretisation in the presence of curvature, it is useful to express the correction in terms of the respective parame- 


\section{Page 21 of 30}

$$
\mu_{\mathrm{opt}} \approx 0.3
$$

For larger values $\mu>\mu_{\mathrm{opt}}$ the diffusive effect introduces a significant reduction in peak density and smearing out of the line profile, whereas for $\mu<\mu_{\mathrm{opt}}$ the violation of $\operatorname{div} \boldsymbol{\alpha}^{I I}=0$ is still appreciable. In Fig. $7(\mathrm{~b})$ one can observe the mending effect of the correction term.

In Fig. 8 the temporal evolution of the sum of the mean absolute value of $\operatorname{div} \boldsymbol{\alpha}^{I I}$ is shown for different correction methods. Relaxation of the system along the dislocation line proves the most effective method, however, carrying out the relaxation to achieve the shown accuracy requires between 10 and 30 relaxation steps after each time step, which causes an increase of the overall computation time by a factor of more than 10. Evaluating the diffusive correction, on the other hand, can be done 'on the fly' and does not cause a notable increase in computation time. For a geometry factor of $\mu=0.3$, we observe an efficient supression of loop fragmentation.

We finally note that the same considerations as above also hold for the shrinkage of a loop. In this case the diffusion coefficient becomes negative which allows the loop to localise.

\section{Application to Microbending}

To investigate the performance of our numerical scheme in a physically relevant situation, we consider the problem of micro-bending of a free-standing thin film. This problem has several interesting features: (i) One is dealing with an intrinsically heterogeneous deformation state where the 'composition' of the dislocation arrangement changes over time due to the growing strain gradients; (ii) These strain gradients are associated with pronounced size effects that have been studied extensively using various dislocation-based models (see e.g. [9], [10], [23], [26]); (iii) the presence of an elastic core at the centre of the specimen leads to the spontaneous emergence of large curvatures in the dislocation system, and hence provides 


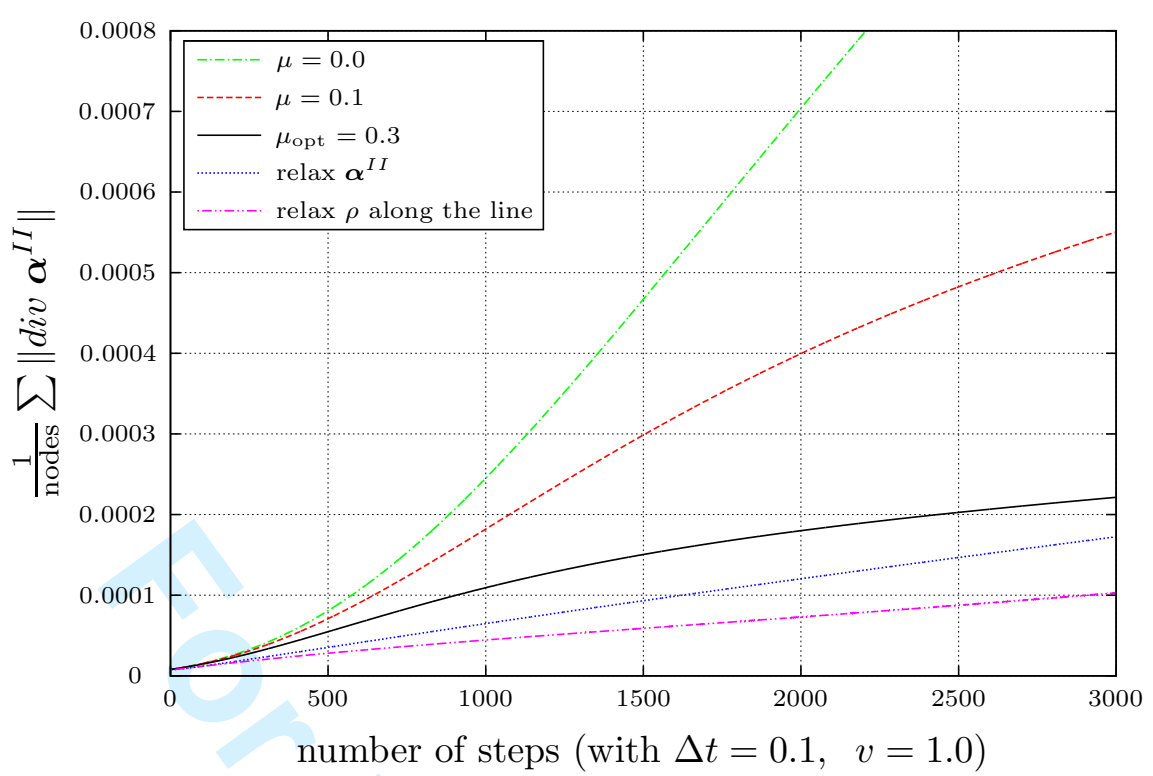

Figure 8. Temporal evolution of the sum of the absolute values of div $\boldsymbol{\alpha}^{I I}$ for different diffusive correction terms. Ideally, the sum would be zero for all steps. Due to the numerical approximation, however, it is non-zero right from the beginning and always increases. A larger amount of diffusion decreases the sum of $\|$ div $\boldsymbol{\alpha}^{I I} \|$ by comparison. The optimum amount of diffusion obtained for $\mu_{\mathrm{opt}}$ is a trade-off between minimizing $\|$ div $\boldsymbol{\alpha}^{I I} \|$ and maintaining the correct peak density value. Relaxation of $\rho$ yields the best result but at a very high computational cost.

an interesting testing case for the numerical accuracy of our computations in a situation where all terms in the evolution equations (38) and (39) yield appreciable contributions to the evolution of the dislocation system.

\subsection{Model Geometry and Stress State}

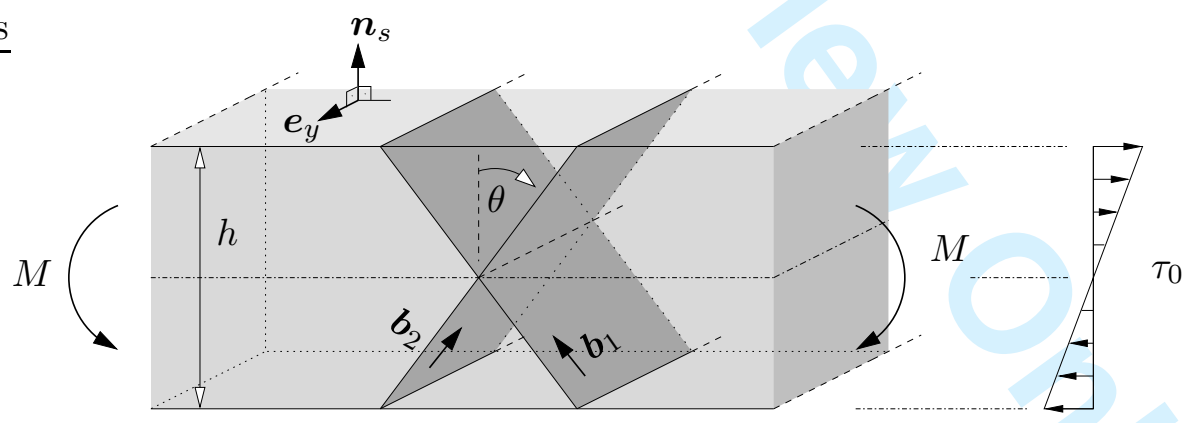

Figure 9. Microbending simulations: Investigated slip geometry. $\tau_{0}$ is the bending stress (resolved shear stress in the slip systems) in the absence of plastic deformation.

We consider bending of a free standing thin film of thickness $h$. The normal vector of the free surfaces is denoted by $\boldsymbol{n}_{\mathrm{s}}$, and we assume without loss of generality that $\boldsymbol{n}_{\mathrm{s}}$ is perpendicular to the $y$ axis of a Cartesian coordinate system. The dimensions of the film in the directions perpendicular to $\boldsymbol{n}_{\mathrm{s}}$ are assumed large so that the system can be considered homogeneous in these directions. The film is deformed by bending around an axis parallel to the $y$ direction. Plastic deformation by dislocation glide may occur on two slip systems that are symmetrically inclined with 


\section{Page 23 of 30}

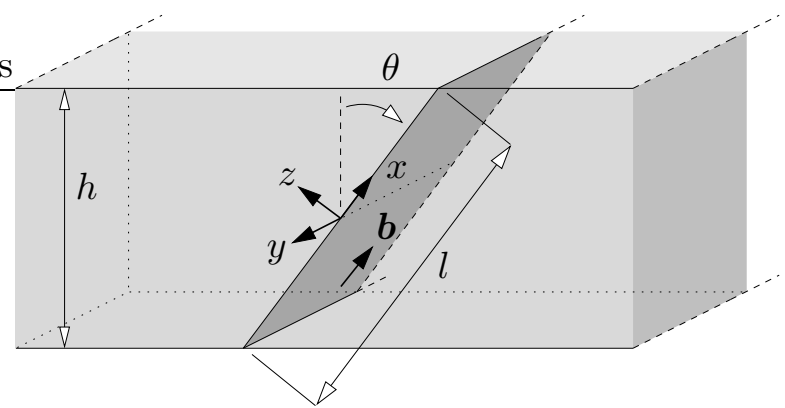

Figure 10. Coordinate system used in our simulations for one of the slip systems: the system is assumed to have infinite extension in $y$ direction.

respect to $\boldsymbol{n}_{\mathrm{s}}$ as shown in Figure 9. The Burgers vector of each slip system is perpendicular to the $y$ axis, defining a plane-strain geometry. Since both slip systems are equivalent, it is sufficient to analyse the evolution of the dislocation system for only one of them. Furthermore, we may restrict ourselves to the evolution of strain and dislocation density on a single slip plane since the system is homogeneous in the directions parallel to the film. We define a Cartesian coordinate system such that the slip plane coincides with the plane $z=0$ as shown in Figure 10 and the origin is located on the neutral fiber in the center of the film. The Burgers vector of the considered slip system points in the positive $x$ direction.

The shear stress in the considered slip system can be formally envisaged as the sum of a reference shear stress $\tau_{0}$ which describes the stress state in a film that is bent to the same radius of curvature $R$ in a purely elastic mannner, and a shear stress $\tau_{1}$ related to the plastic strain $\gamma$, which is the sum of the plastic strains from both slip systems. The stress $\tau_{0}$ is given by

$$
\tau_{0}(x)=\frac{\sin (2 \theta) h}{R(1-\nu)} G \frac{x}{l}
$$

where $G$ is the shear modulus of the material, $\nu$ is Poisson's number, and $l$ is the film width projected on the $x$ direction (Figure 10). For the geometry under consideration, plastic deformation of a fiber leads to a stress reduction that is proportional to the axial strain. Assuming isotropic material properties and making the standard assumption that straight specimen cross sections (cross sections parallel to the bending axis) remain straight during bending, we find that

$$
\tau_{1}(x)=-\frac{\sin ^{2}(2 \theta)}{1-\nu} G \gamma(x)
$$

where we have accounted for the plastic strain contributions from both slip systems. The bending moment $M$ (moment per unit length in the $y$ direction) corresponding to a given bending radius $R$ and plastic deformation state is evaluated from the stresses $\tau_{0}$ and $\tau_{1}$ as

$$
\frac{M}{h^{2}}=\frac{4}{\sin (2 \theta)} \int_{0}^{1 / 2}\left(\tau_{0}(u)+\tau_{1}(u)\right) u \mathrm{~d} u
$$

where $u=x / l$ and $\tau_{0,1}(u):=\tau_{0,1}(x=u l)$. The scaled bending moment $M / h^{2}$ in the following serves as measure of the characteristic stress in our bending simulations. 


\subsection{Constitutive relations}

We use a linear-viscous model of overdamped dislocation motion by assuming that the dislocation velocity depends linearly on the difference between the local shear stress $\tau$ and a local yield stress $\tau_{\mathrm{y}}$ :

$$
v= \begin{cases}\frac{b}{B}\left(\tau-\tau_{\mathrm{y}}\right) & \text { if } \tau>+\tau_{\mathrm{y}} \\ \frac{b}{B}\left(\tau+\tau_{\mathrm{y}}\right) & \text { if } \tau<-\tau_{\mathrm{y}} \\ 0 & \text { otherwise }\end{cases}
$$

Physically, the yield stress on a given slip system is governed by the intersection of moving dislocations with 'forest' dislocations of other systems, leading to junction formation. In the present case, both slip systems are equivalent, hence we can relate the yield stress to the dislocation density on the slip system itself. We assume a Taylor relationship,

$$
\tau_{\mathrm{y}}(x)=a G b \sqrt{\rho(x)},
$$

where $a \approx 0.4$ is a nondimensional constant characterising the strength of dislocation-forest interactions.

In our constitutive model we neglect effects of large-scale dislocation curvature on the dislocation velocity (line tension as considered in $[23,26]$ ). We also neglect back stresses resulting from the 'piling up' of dislocations of the same sign [26, 27], which are also implemented in DDD simulations of microbending ([24], [25]). . Consistent with our assumption that dislocation motion is controlled by forest interactions, we do not impose specific boundary conditions to account for image effects at the free surfaces. Instead, we allow dislocations of all orientations to enter or leave freely, simply extrapolating the dynamics inside the film across the boundary (similar to the 'natural' outflow boundary conditions in [24], where contrary to our boundary conditions - only outflow and no inflow of dislocations from the surface was allowed). In physical terms our boundary conditions imply that we assume that surface sources have no activation stress other than the yield stress that controls near-surface dislocation motion inside the sample.

\subsection{Results}

As initial condition for our simulated bending tests, we assume an isotropic dislocation pattern with zero mean curvature and space-independent total dislocation density $\rho=10^{13} \mathrm{~m}^{-2}$, i.e., initially dislocations of all orientations are present with equal density $\rho(x, \varphi)=(1 / 2 \pi) \times 10^{13} \mathrm{~m}^{-2}$. This initial condition describes a homogeneous and statistically isotropic arrangement of straight dislocation lines threading the film at random points. We carry out quasistatic simulated bending tests by increasing the load $\tau_{0}(x)$ in small steps. In physical terms, this corresponds to slowly decreasing an imposed curvature radius on the thin film. We start from the critical stress level where the shear stress at the surface reaches the yield stress corresponding to the initial dislocation density. After each stress increment, we solve the equations of motion while simultaneously evaluating the increase of the plastic strain and the concomitant decrease of local stresses and increase in flow stress. Due to these changes in the internal stress state the strain rate gradually decreases towards zero. We trace this relaxation until the maximum strain rate has everywhere dropped below a prescribed low level and then record the bending moment $M$ and strain profile $\gamma(x)$ as well as the dislocation and curvature patterns 


\section{Page 25 of 30}

$\rho(x, \varphi)$ and $k(x, \varphi)$ before applying the next stress increment.
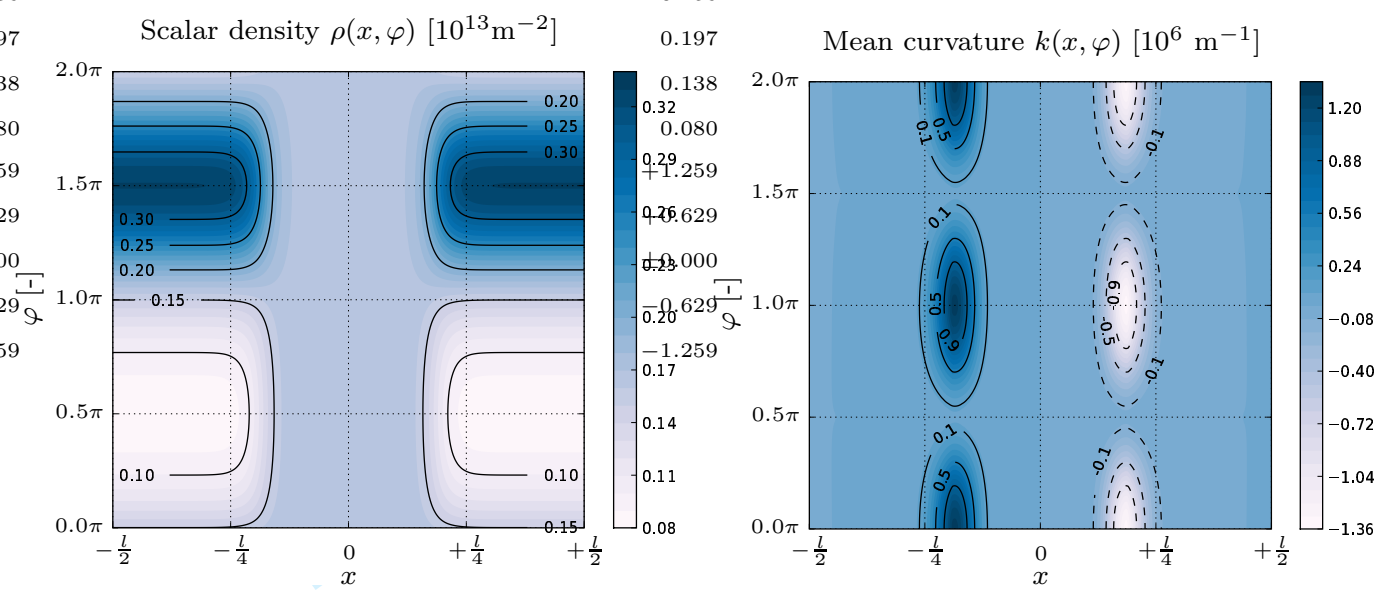

Figure 11. Dislocation density and curvature patterns in $(x, \varphi)$ configuration space for a film thickness of $h=3 \mu \mathrm{m}$ and bending moment (per unit length in $y$ ) $M=2 \cdot 10^{-15} \mathrm{GPa} \mathrm{m}^{2}$.

The following examples were computed using finite differences with an equidistant mesh. The resolution was about 700 spatial nodes and 100 angular nodes; a fifth-order Runge-Kutta method with adaptive step size control turned out to be an effective time integration scheme: the computational cost on a $2.4 \mathrm{GHz}$ quad core work station for each of the following simulations is about 20 minutes.

Typical density and curvature patterns are shown in Fig. 11. These patterns are characterised by the presence of an elastic core region where the stress $\tau_{0}(x)$ is less than the yield stress corresponding to the initial dislocation density. No deformation activity takes place within this region and, hence, the dislocation density and curvature remain at their initial values. Curvature spatially localizes near the boundaries of the elastic core region which are moving inwards with increasing stress. In terms of the orientation coordinate, curvature is strongest for near-screw orientations while edge dislocations are only weakly bent. Density accumulates in the $\varphi=3 \pi / 2$ direction, which is the orientation of edge dislocations required to accomodate the bending strain gradient. At the same time, the density of dislocations of other orientations decreases. The decrease is most pronounced for the $\varphi=\pi / 2$ orientation, i.e. for edge dislocations of the opposite sign.

As the strain increases, we observe that the total dislocation density in the plastic regions increases strongly, as shown in Fig. 12. This increase goes along with an increase of the $\alpha_{11}$ component of the Kröner-Nye tensor, i.e. accumulation of 'geometrically necessary' dislocations required to accomodate the increasing plastic strain gradients around the narrowing central elastic core. This is illustrated in Fig. 13.

The increase of total dislocation density that comes with the accumulation of geometrically necessary dislocations leads, according to the Taylor relation, to an increase in flow stress. As strain gradients are bigger in smaller specimens and therefore the accumulation of geometrically necessary dislocations is more pronounced, this leads to a size dependent hardening. This is illustrated in Fig.14 which shows the scaled bending moment $M / h^{2}$ as a function of the average plastic strain $\langle\gamma\rangle=(1 / h) \int_{-h / 2}^{+h / 2}|\gamma(x)| \mathrm{d} x$. While the initial flow stress is size independent, the hardening rate and the flow stress at finite strain increase with decreasing film thickness. 

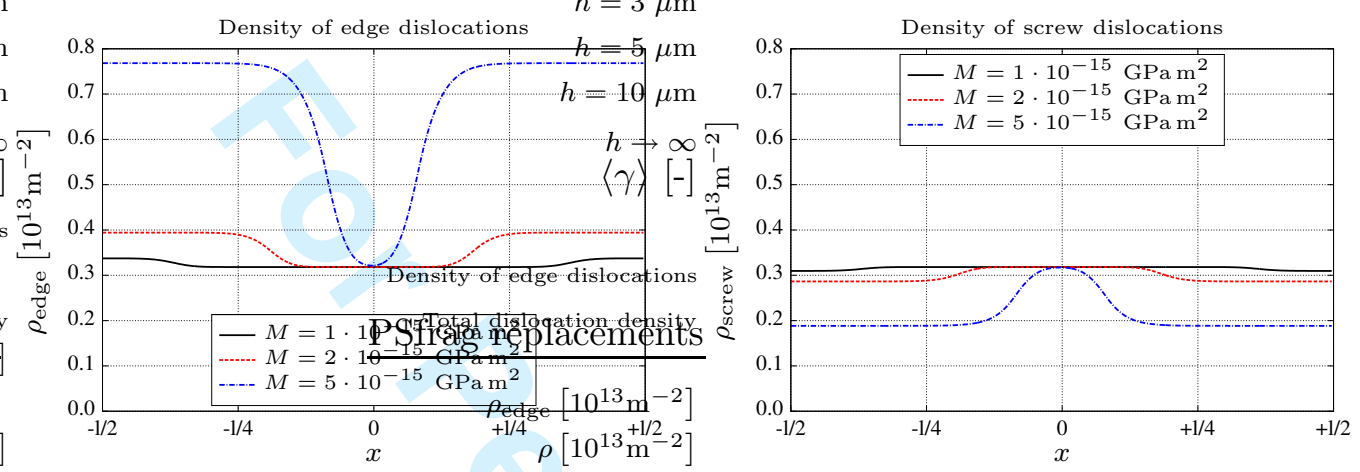

Figure 12. The top diagram shows the total dislocation density $\rho(x)=\int_{0}^{2 \pi} \rho(x, \varphi) \mathrm{d} \varphi$. The left and right diagrams show the densities of edge and screw dislocation for $h=3 \mu \mathrm{m}: \rho_{\text {edge }}=\rho(\varphi=0.5 \pi)+\rho(\varphi=1.5 \pi)$ and $\rho_{\text {screw }}=\rho(\varphi=0)+\rho(\varphi=\pi)$.
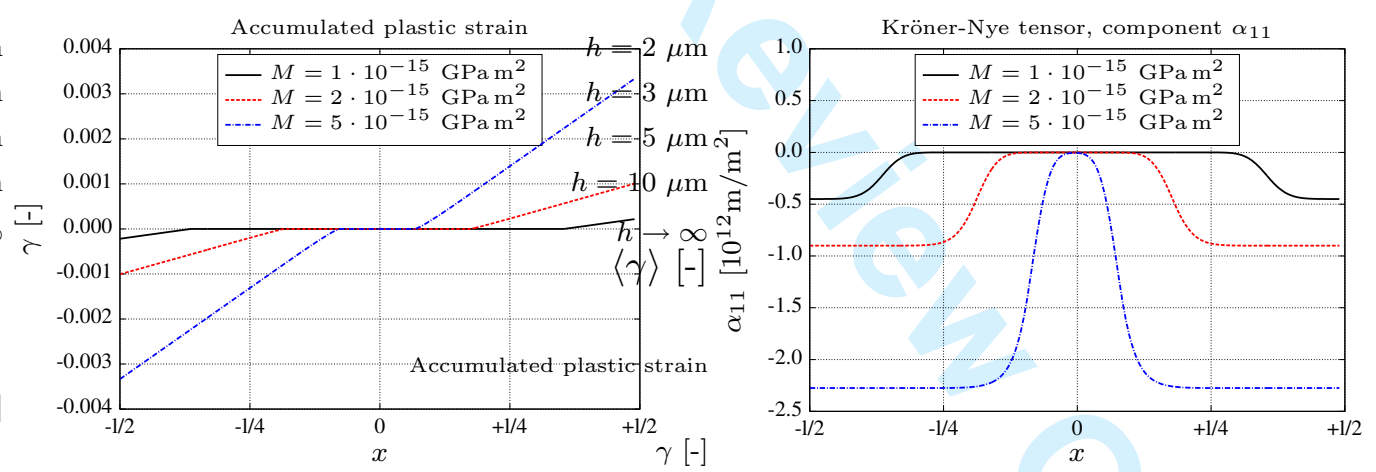

Figure 13. Accumulated plastic strain and (11) component of the Kröner-Nye tensor $\alpha$ for $h=3 \mu \mathrm{m}$ and different bending moments

\subsection{Discussion}

An often-repeated argument for analyzing this type of size-dependent behavior (see e.g. [9]) runs as follows: The dislocation density in a material is the sum of a geometrically necessary density $\rho_{\mathrm{G}}$ (up to a geometrical factor $\eta$ equal to the strain gradient divided by the Burgers vector modulus) and a so-called 'statistically stored' density $\rho_{\mathrm{S}}$. (The latter is in fact nothing but the total dislocation density minus the geometrically necessary one, but the argument treats it like an independent physical variable.) From Taylor's relation for the flow stress one 


\section{Page 27 of $30 \quad$ Philosophical Magazine \& Philosophical Magazine Letters}

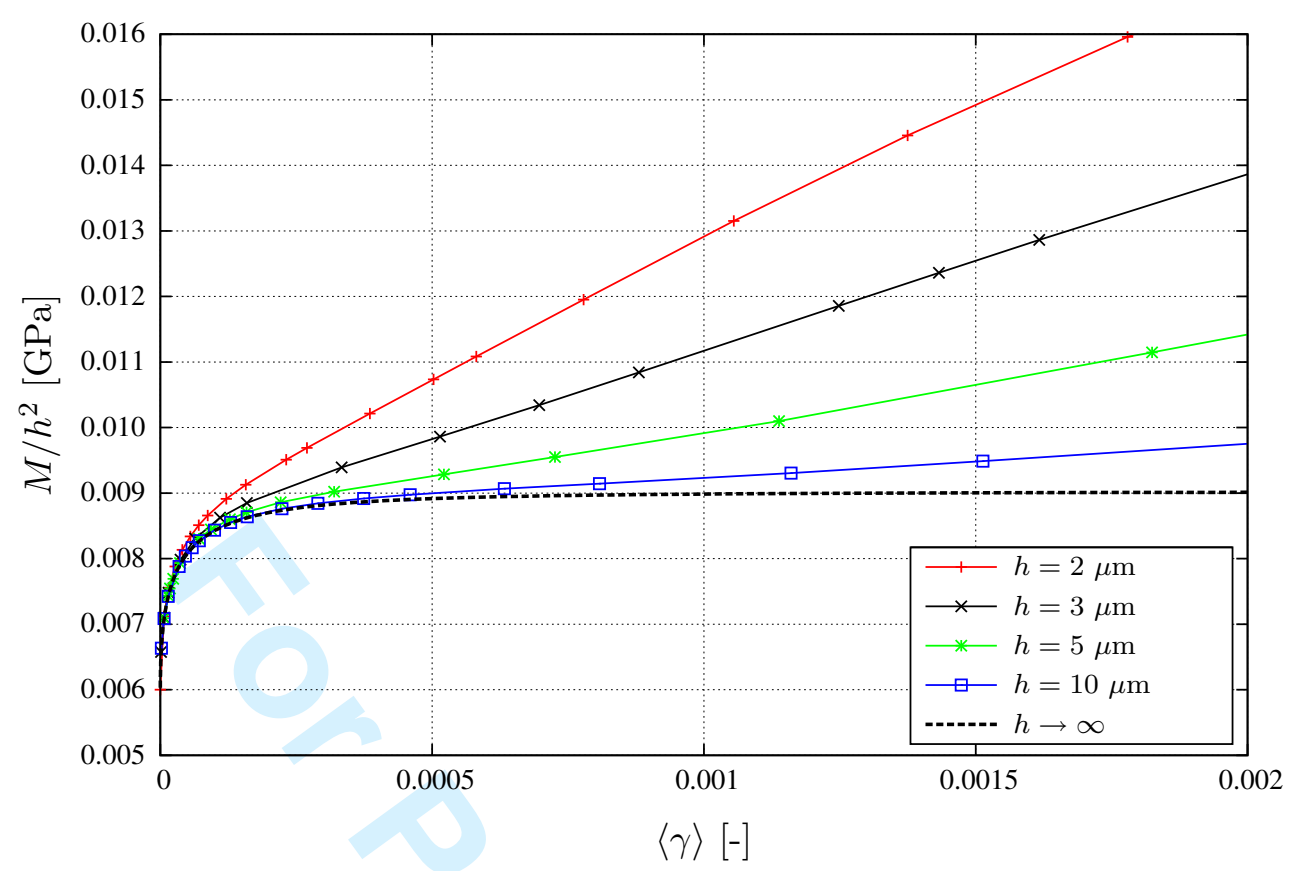

Figure 14. Bending curves for various film thicknesses; the curve for infinite thickness has been calculated by assuming ideal plasticity and using the standard assumption of straight specimen cross-sections.

obtains

$$
\tau_{\mathrm{y}}=a G b \sqrt{\rho}=a G b \sqrt{\rho_{\mathrm{S}}+\rho_{\mathrm{G}}}=a G b \sqrt{\rho_{\mathrm{S}}+\frac{\eta}{b}|\nabla \gamma|} .
$$

One then makes the crucial assumption that the strain dependence of the 'statistically stored' density $\rho_{\mathrm{S}}(\gamma)$ does not depend on the presence or absence of strain gradients. Estimating the characteristic strain gradient as $\langle\gamma\rangle / L$ where $\langle\gamma\rangle$ is a characteristic strain and $L$ a characteristic dimension of the specimen then leads to a scaling relation for the characteristic flow stress $\tau$ :

$$
\left[\tau_{\gamma, L}\right]^{2}=\left[\tau_{\gamma, \infty}\right]^{2}+a^{2} G^{2} \eta b \frac{\langle\gamma\rangle}{L}
$$

where $\tau_{\langle\gamma\rangle, \infty}=a G b \sqrt{\rho_{\mathrm{S}}(\gamma)}$. To test this scaling relation we have to translate the notations to our problem. The characteristic dimension $L$ corresponds to the film thickness $h$, and the macroscopic flow stress corresponds to the scaled bending moment $M / h^{2}$. As strain measure we use the average plastic strain $\langle\gamma\rangle$ as defined above. The infinite-system limit is obtained by solving the bending problem for an ideally plastic material with flow stress corresponding to the initial dislocation density. The corresponding deformation curve is denoted as $\left[M_{\langle\gamma\rangle} / h^{2}\right]_{\infty}:=\lim _{h \rightarrow \infty}\left[M_{\langle\gamma\rangle, h} / h^{2}\right]$. The total dislocation density $\langle\rho\rangle$ in the specimen is obtained by averaging $\rho(x, \varphi)$ over the specimen cross section and integrating over all orientations $\varphi$, and the 'geometrically necessary' density $\left\langle\rho_{\mathrm{G}}\right\rangle$ is obtained as the minimum density of dislocations on each of the two slip system required to accomodate the plastic strain gradient: We evaluate the component $\alpha_{11}$ of the dislocation density tensor, average this over the specimen cross section, divide by $b$ and multiply with the geometrical factor $1 /(2 \sin \theta)$. 
If the above argument would apply to our simulations, a plot of $\left[M_{\langle\gamma\rangle, h} / h^{2}\right]^{2}-$ $\left[M_{\langle\gamma\rangle} / h^{2}\right]_{\infty}^{2}$ versus the scaled plastic strain $\langle\gamma\rangle b / h$ should yield a straight line passing through the origin. The same should be true for a plot of the total dislocation density $\langle\rho\rangle$ (reduced by the infinite-system value) versus the scaled plastic strain. Our simulations, however, indicate a different behavior. Even though the 'geometrically necessary' dislocation density increases approximately linearly with scaled plastic strain, the same is not true for the total dislocation density: the increase in total density initially lags behind the increase in the 'geometrically necessary' density and only catches up at large strains (Fig. 15). As a consequence, the scaling plot of the reduced bending moment vs. the scaled plastic strain (Fig. 16) fails to produce a straight line.

What are the reasons for the discrepancy between the simulation results and the naive scaling argument? Our investigation of dislocation density evolution indicates that the linear increase of the 'geometrically necessary' density with reduced strain is not matched by a concomitant increase of the total density, and hence of the flow stress. The reason is that we allow dislocations to change their orientation, besides freely entering or leaving through the specimen surface. As a consequence, part of the increase of the 'geometrically necessary' density is not produced by additional dislocations entering through the surfaces, but rather by changes in orientation of existing dislocations and by the loss of dislocations of the 'wrong' sign. As a consequence, the total dislocation density increases, at least for small strains / large system sizes, much less than expected according to the simple scaling argument. This indicates that the assumption of a separate 'statistically stored' density that evolves in a size-independent manner is not warranted.

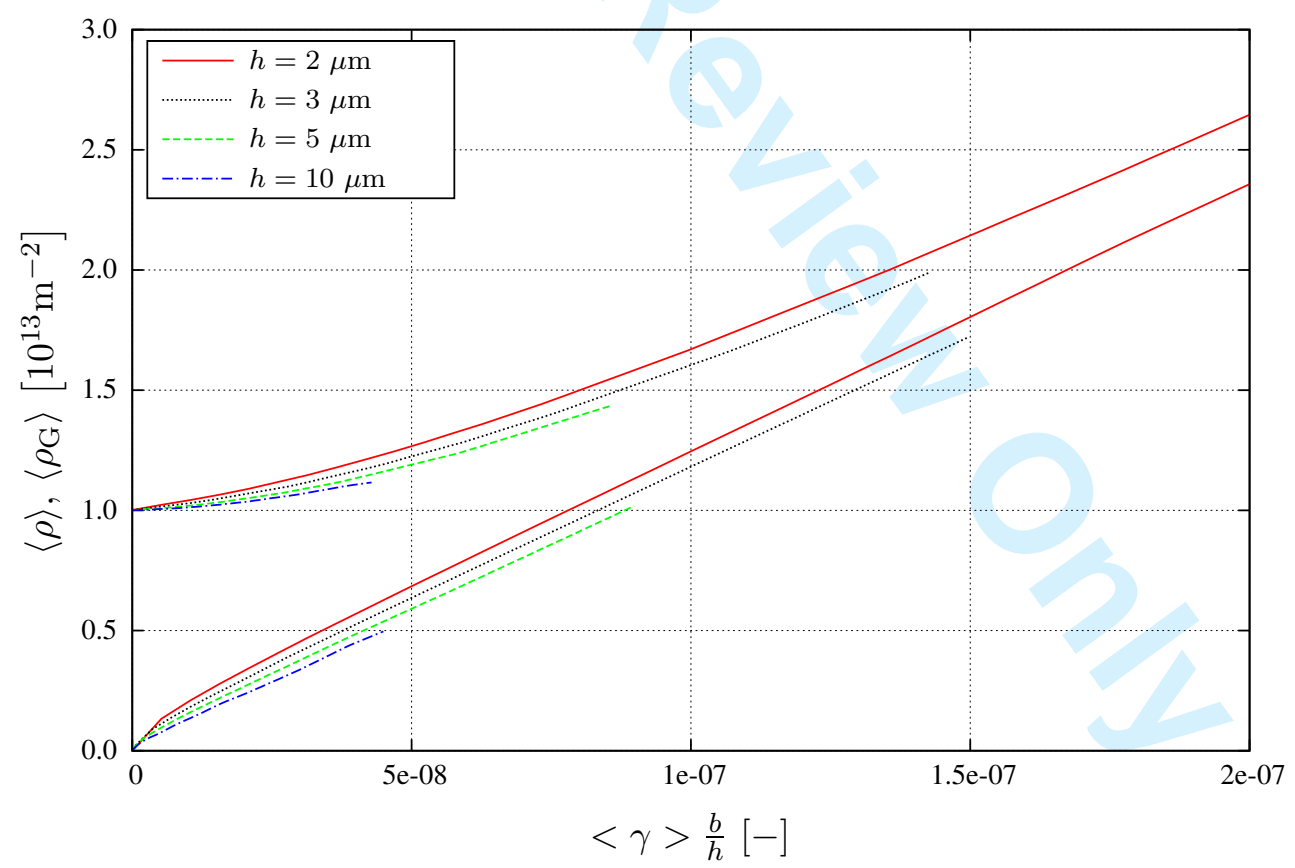

Figure 15. Scaling plot of the total and 'geometrically necessary' dislocation density vs reduced strain $\langle\gamma\rangle b / h$. The upper line group is the plot for $\langle\rho\rangle$, the lower line group is the plot for $\left\langle\rho_{\mathrm{G}}\right\rangle$. 


\section{Page 29 of 30}

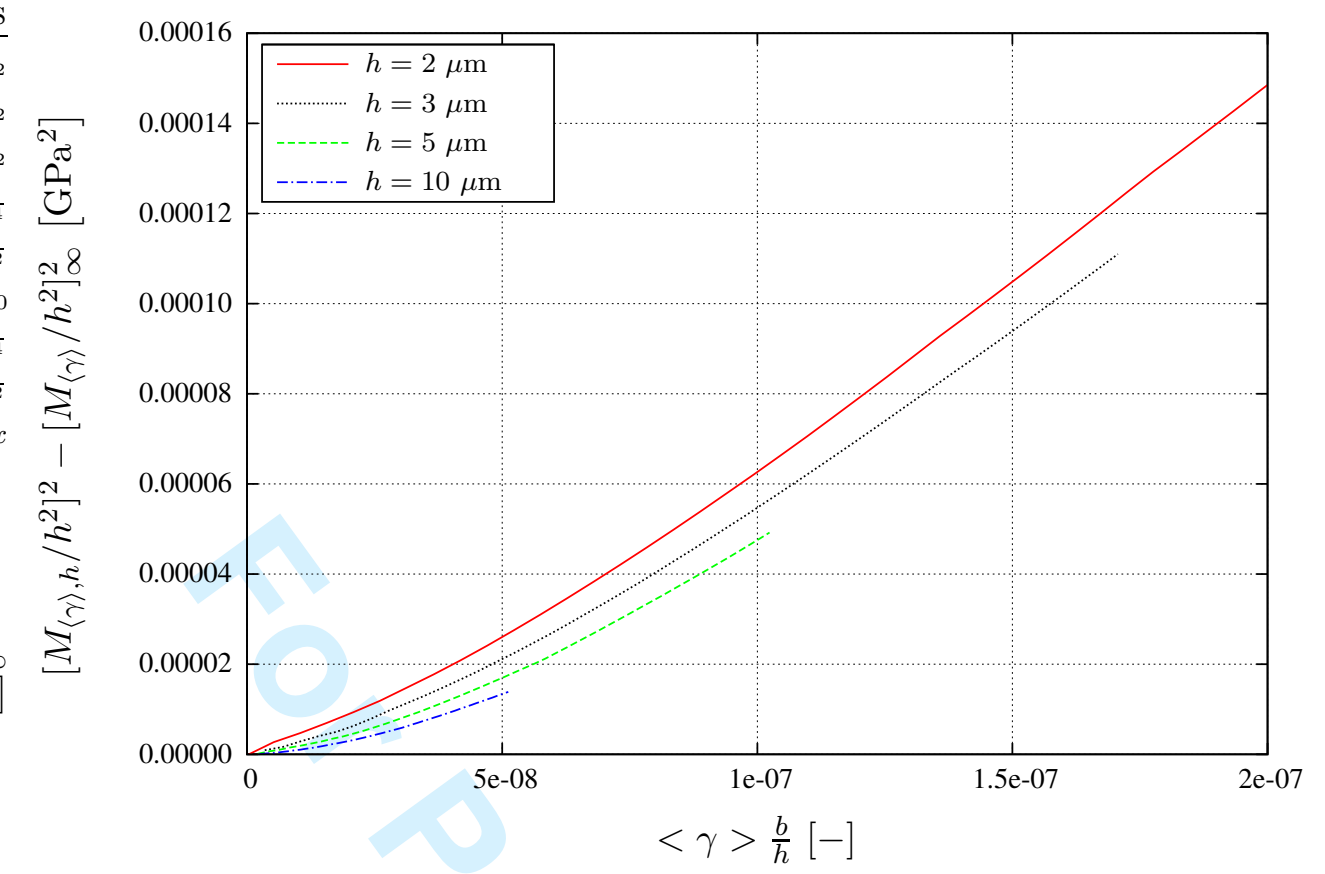

Figure 16. Scaling plot of the bending curves: $\left[M_{\langle\gamma\rangle, h} / h^{2}\right]^{2}-\left[M_{\langle\gamma\rangle} / h^{2}\right]_{\infty}^{2}$ vs reduced strain $\langle\gamma\rangle b / h$.

\section{Conclusion and Outlook}

We demonstrated that ECT as a generalisation of the classical Kröner theory can capture several fundamental phenomena of the evolution of systems of curved dislocations which cannot be recovered from other dislocation based continuum theories. In particular, the simple benchmark problem of an ensemble of expanding loops showed that the averaging process underlying ECT conserves important kinematic properties of dislocation loops. Studying the expansion of a single quasi-discrete loop served to elucidate certain numerical problems arising from the discretization of orientation space and to find appropriate remedies. We then demonstrated that the evolution of dislocation densities in orientation space may be a key factor in the description of inhomogeneous deformation processes, as changes in dislocation orientation may be an important mechanism for providing the 'geometrically necessary' dislocations needed to accomodate strain gradients. Accordingly, simulations of microbending using a simple Taylor-based flow rule yielded results that differ substantially from the predictions of gradient plasticity theories that assume strain gradients to be accomodated exclusively by the introduction of additional excess dislocations.

The present theory offers a versatile tool for treating the evolution of dislocation systems without having to resolve the discrete dynamics of all dislocation lines, or having to make arbitrary assumptions regarding the 'composition' of the dislocation arrangement. Rather, the distribution of dislocations over the various orientations follows naturally from the theory once the basic constitutive relations have been specified which relate the dislocation velocity to the dislocation pattern. In our simulations of microbending we used a most simple constitutive law, assuming local validity of the Taylor relationship but neglecting non-local contributions to the flow stress that may be associated with line curvature and/or with the piling up of dislocations of the same sign [26]. Such contributions can be easily included 
and their influence will be investigated in future work, which will also provide an extensive comparison with the results of discrete simulations.

\section{Acknowledgement}

Financial support of the European Commission under NEST-2005-PATH-COM043386 is gratefully acknowledged.

\section{References}

[1] E. KRÖNER, Kontinuumstheorie der Versetzungen und Eigenspannungen (Springer, 1958).

[2] J. NyE, Acta Metall. 1, 153-162 (1953).

[3] B. A. Bilby, R. Bullough, and E. Smith, Proc. Roy. Soc. London Ser. A 231, 263-273 (1955).

[4] K. Kondo, Proc. 2. Japan Nat. Congress of Appl. Mech. pp. 41-47 (1952).

[5] A. Acharya, J. Mech. and Phys. Solids 49(4), 761-784 (2001).

[6] A. Acharya, Proceedings: Mathematical, Physical and Engineering Sciences 459(2034), 1343-1363 (2003).

[7] S. N. Varadhan, A. Beaudoin, A. Acharya, and C. Fressengeas, Modelling Simul. Mater. Sci. Eng. 14, 1245-1270 (2006).

[8] R. Sedláček, J. Kratochvíl, and E. Werner, Phil. Mag. 83(31-34), 3735-3752 (2003).

[9] H. Gao, Y. Huang, and W. E. A. Nix, J. Mech. and Phys. Solids 47, 1239-1263 (1999).

[10] M. Zaiser and E. C. Aifantis, Scripta Mater. 48, 133-139 (2003).

[11] I. Groma, Phys. Rev. B 56(10), 5807-5813 (1997).

[12] M. Zaiser, M. C. Miguel, and I. Groma, Phys. Rev. B 64(Nov), 224102 (2001).

[13] I. Groma, F. Csikor, and M. Zaiser, Acta Mater. 51, 1271-1281 (2003).

[14] A. Kosevich Dislocations in solids vol.1. the elastic theory 1, 33-141 (1979).

[15] A. El-Azab, Phys. Rev. B 61(18), 11956-11966 (2000).

[16] T. Hochrainer, M. Zaiser, and P. Gumbsch, Phil. Mag. 87(8-9), 1261-1282 (2007).

[17] A. Acharya, A. Roy, and A. Sawant, Scripta Mater. 54, 705-710 (2005).

[18] T. Hochrainer, Evolving systems of curved dislocations: Mathematical foundations of a statistical theory, PhD thesis, University of Karlsruhe, IZBS, 2006; Shaker Verlag, Aachen 2007.

[19] A. Harten, J. Comput. Phys. 71(2), 231-303 (1987).

[20] J. C. Strikwerda, Finite difference schemes and partial differential equations, 2nd edition (SIAM, 2004).

[21] R. Sedláček, C. Schwarz, J. Kratochvíl and E. Werner, Phil. Mag. 87, 1225-1260 (2007).

[22] K. W. Morton, Numerical solution of convection diffusion problems, 1st. edition (Chapman \&3 Hall, 1996).

[23] R. SEdláčEK, Mater. Sci. Eng. A 393, 387-395 (2005).

[24] S. Yefimov, E. Van der Giessen And I. Groma, Modelling Simul. Mater. Sci. Eng. 12, 1069-1086, (2004)

[25] Cleveringa, H.H.M. and Van der Giessen, E. And Needleman, A.Int. J. Plasticity 15 837-868, (1999)

[26] M. Zaiser, N. Nikitas, T. Hochrainer, and E.C. Aifantis, Phil. Mag. 87, 1283-1306 (2007).

[27] M. ZAiser and T. HOChrainer, Scripta Mater. 54, 717-721 (2006). 